\title{
A CAYLEY-DICKSON PROCESS FOR A CLASS OF STRUCTURABLE ALGEBRAS
}

BY

\author{
B. N. ALLISON ${ }^{1}$ AND J. R. FAULKNER ${ }^{2}$
}

\begin{abstract}
In this paper, we study the class of all simple structurable algebras with the property that the space of skew-hermitian elements has dimension 1. These algebras with involution have arisen in the study of Lie algebra constructions. The reduced algebras are isotopic to $2 \times 2$ matrix algebras. We study a Cayley-Dickson process for rationally constructing some algebras in the class including division algebras and nonreduced nondivision algebras. An important special case of the process endows the direct sum of two copies of a 28-dimensional degree 4 central simple Jordan algebra $\mathscr{B}$ with the structure of an algebra with involution. In preparatory work, we obtain a procedure for giving the space $\mathscr{B}_{0}$ of trace zero elements of any such Jordan algebra $\mathscr{B}$ the structure of a 27 -dimensional exceptional Jordan algebra.
\end{abstract}

The 56-dimensional irreducible module $\mathfrak{T}$ for the split simple Lie algebra of type $E_{7}$ over a field $\mathfrak{f}$ possesses the structure of an algebra with involution that can be used in the construction of the split simple Lie algebras of type $E_{7}$ and $E_{8}$ [2,5 and 11]. In order to study nonsplit Lie algebras of these types, it is of interest to have a rational (in the sense that base field extension is not involved) construction of $\mathfrak{f}$-forms of $\Re_{\bar{f}}$, where $\overline{\mathfrak{f}}$ denotes the algebraic closure of $\mathfrak{f}^{\prime}$ and a $\mathfrak{f}^{-}$-form of $\Re_{\bar{f}}$ is an algebra with involution $\left(\mathscr{Q},{ }^{-}\right)$over $\mathfrak{H}^{\mathfrak{t}}$ such that $\left(\mathscr{Q},{ }^{-}\right)_{\overline{\mathrm{f}}}^{-} \cong \mathfrak{K}_{\overline{\mathrm{f}}}$. The underlying vector space for $\Re$ is the vector space of all $2 \times 2$ matrices with scalar diagonal entries and nondiagonal entries chosen from the 27-dimensional split exceptional central simple Jordan algebra. The multiplication on $\mathfrak{N}$ is strikingly similar to the multiplication on the split octonion algebra $\theta$ over $\mathfrak{t}$ as obtained from the Zorn construction [9, p. 142]. Thus, it is natural to ask whether there is a Cayley-Dickson process for constructing ${ }^{-}$-forms $\Re_{-}$that is analogous to the classical Cayley-Dickson process

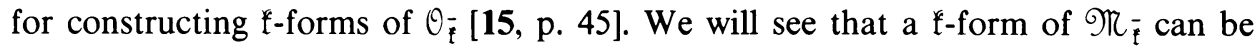
constructed by endowing the direct sum of two copies of a 28-dimensional degree 4 central simple Jordan algebra with the structure of an algebra with involution (see Example 6.12). This construction is a special case of the Cayley-Dickson process that is the main subject of this paper.

In [1], a class of algebras with involution called structurable algebras was studied. The matrix algebra $\mathfrak{N}$ referred to in the previous paragraph is an example of a

Received by the editors April 1, 1983.

1980 Mathematics Subject Classification. Primary 17A30; Secondary 17B60, 17C20.

${ }^{1}$ Research supported in part by an NSERC Grant.

${ }^{2}$ Research supported in part by an NSF Grant. 
simple structurable algebra $\left(\Theta,,^{-}\right)$such that space $\delta\left(\Theta,{ }^{-}\right)=\left\{x \in \mathscr{U}^{\prime} \mid \bar{x}=-x\right\}$ has dimension 1 . In $\$ \S 1-4$, we investigate the class of all simple structurable algebras $\left(Q,,^{-}\right)$such that $\delta\left(Q,,^{-}\right)$has dimension 1 . Any such algebra $\left(Q,,^{-}\right)$possesses a quartic form $\nu$ that occurs naturally as the denominator in the inversion operation. This quartic form gives $\left(Q,,^{-}\right)$the structure of a Freudenthal triple system and thereby allows us to make use of earlier work of Ferrar on such systems.

The Cayley-Dickson process studied in this paper has as its starting point a Jordan algebra possessing a Jordan norm of degree 4. These norms are studied in $\$ 5$. An example of a Jordan norm of degree 4 is the generic norm on a degree 4 separable Jordan algebra. We obtain as a consequence of our work in $\$ 5$ the fact that the space of trace 0 elements in a degree 4 separable Jordan algebra has the structure of a degree 3 separable Jordan algebra. In particular, starting with a 28-dimensional central simple degree 4 (special) Jordan algebra, a 27-dimensional exceptional central simple Jordan algebra is obtained.

In $\$ 6$ we describe the Cayley-Dickson process that produces simple structurable algebras $\left(Q,,^{-}\right)$such that $\operatorname{dim}_{\mathfrak{f}} \delta\left(Q,,^{-}\right)=1$. In $\S 7$, we show that for certain choices of the parameters the process produces division algebras. We also discuss in $\S 7$ the use of such algebras in the construction of exceptional central simple Lie algebras over a field of characteristic zero. Finally, in $§ 8$, we give necessary and sufficient conditions for the process to give reduced algebras.

Before proceeding, we fix some conventions and notation. Throughout the paper, we assume that $\mathfrak{f}$ is field of characteristic $\neq 2$ or 3 . All vector spaces and algebras over $\mathfrak{f}$ are assumed to be finite dimensional over $\mathfrak{k}$. Algebras are not necessarily assumed to be associative and (except for Lie algebras) all algebras are assumed to possess a multiplicative identity denoted by 1 . If $\mathbb{Q}$ is an algebra and $x, y, z \in \mathbb{Q}$, we write $[x, y, z]=(x y) z-x(y z)$. An algebra with involution $\left(\Theta,^{-}\right)$is an algebra $Q$ together with an anti-isomorphism $x \rightarrow \bar{x}$ of period two. If $\left(Q,,^{-}\right)$is an algebra with involution, we denote the spaces of hermitian and skew-hermitian elements by

$$
\mathcal{H}\left(\mathbb{Q},,^{-}\right)=\{x \in \mathscr{Q} \mid \bar{x}=x\} \text { and } S\left(\mathscr{Q},{ }^{-}\right)=\{x \in \mathscr{Q} \mid \bar{x}=-x\} \text {, }
$$

in which case $\mathbb{Q}=\mathcal{H}\left(\mathcal{Q},{ }^{-}\right) \oplus S\left(\mathbb{Q},{ }^{-}\right)$.

Finally, suppose $\mathcal{V}$ is a vector space over $\mathfrak{t}$ and $m$ is a nonnegative integer $\leqslant 4$. A form of degree $m$ is a function $F: \mathcal{V} \rightarrow \mathfrak{f}$ such that, relative to some choice of basis for $\mathfrak{V}, F$ is induced by a homogeneous polynomial (possibly zero) of degree $m$ in $\operatorname{dim}_{\mathfrak{f}}(\mathcal{V})$ variables over $\mathfrak{t}$. If $F$ is a form of degree $m$ on $\mathscr{T}$ and $K / \mathfrak{f}$ is a field extension then, since $m \leqslant 4<|\mathfrak{f}|, F$ extends uniquely to a form of degree $m$ on $\Upsilon_{K}$. The extension is also denoted by $F$. If $\lambda$ is transcendental over $\mathfrak{f}$ and $F$ is a form of degree $m$ on $\mathcal{V}$, write $F(c+\lambda b) \equiv F(c)+\lambda \partial_{b} F(c)\left(\bmod \lambda^{2}\right)$ for $b, c \in \mathscr{B}$. Then, $\partial_{b} F$ is a form of degree $m-1$ (regarding 0 as the unique form of degree -1 ) and $b \rightarrow \partial_{b} F$ is linear.

The authors wish to express their gratitude to the referee for several helpful suggestions. 
1. Structurable algebras. We collect in this section the facts about structurable algebras that will be used in later sections.

An algebra with involution $\left(Q,,^{-}\right)$over $\mathbb{t}$ is called a structurable algebra if

$$
\left[V_{x, y}, V_{z, w}\right]=V_{V_{x, y, z}, w}-V_{z, V_{v, x} w}
$$

for $x, y, z, w \in \mathbb{Q}$, where $V_{x, y} \in \operatorname{End}_{\mathrm{f}}(\mathfrak{Q})$ is defined by

$$
V_{x, y} z=\{x, y, z\}=(x \bar{y}) z+(z \bar{y}) x-(z \bar{x}) y .
$$

We assume for the rest of the section that $\left(\mathscr{Q},,^{-}\right)$is a structurable algebra over $¥$. We write $\mathcal{S}=\mathcal{S}\left(\mathbb{Q},{ }^{-}\right)$and $\mathcal{H}=\mathcal{H}\left(\mathbb{Q},{ }^{-}\right)$. Define $\psi_{(\mathbb{Q},-)}: \mathbb{Q} \times \mathbb{Q} \rightarrow \mathcal{S}$ by $\psi_{(\mathbb{Q},-)}(x, y)=x \bar{y}$ $-y \bar{x}$. If no ambiguity exists, we write $\psi=\psi_{(\Theta,-)}$. Note that $\psi(s, 1)=2 s$ for $s \in \mathcal{S}$ and so $\psi$ is a surjection. For $x, z \in \mathscr{Q}$, define $U_{x, z}, L_{x}, R_{x}$, and $U_{x} \in \operatorname{End}_{\mathrm{f}}(\mathscr{Q})$ by $U_{x, z} y=V_{x, y} z, L_{x} y=x y, R_{x} y=y x$, and $U_{x}=U_{x, x}$. Then we have the following identities for $x, y \in \mathbb{Q}, s \in \mathcal{S}($ see $[1, \S 1]$, [2, Lemma 2], and [3, §11]):

$$
\begin{gathered}
{[s, x, y]=-[x, s, y]=[x, y, s],} \\
U_{x, y}-U_{y, x}=L_{\psi(x, y)}, \\
V_{x, s y}-V_{y, s x}=-L_{\psi(x, y)} L_{s}, \\
s \psi(x, y) s=-\psi(s x, s y),
\end{gathered}
$$

and

$$
L_{s} U_{x, y} L_{s}=-U_{s x, s y} .
$$

Also $0=\left[V_{x, y}, V_{x, y}\right]=V_{\{x, y, x\}, y}-V_{x,\{y, x, y\}}($ by $(1.1))$ and so

$$
V_{U_{x}, y}=V_{x, U_{y} x}
$$

for $x, y \in \mathbb{Q}$. Thus, if $x \in \mathscr{Q}$ and $s \in \mathcal{S}$,

$$
\begin{aligned}
V_{\{x, s x, x\}, s x} & =V_{x,\{s x, x, s x\}}=-V_{x, s\{x, s x, x\}} \quad(\text { by }(1.6)) \\
& =-V_{\{x, s x, x\}, s x}+L_{\psi(x,\{x, s x, x\})} L_{s} \quad(\text { by }(1.4)) .
\end{aligned}
$$

Therefore,

$$
2 V_{\{x, s x, x\}, s x}=L_{\psi(x,\{x, s x, x\})} L_{s}
$$

for $s \in \mathcal{S}, x \in \mathbb{Q}$.

For $E \in \operatorname{End}_{\mathfrak{f}}(\mathfrak{Q})$, define $E^{\delta}, E^{\varepsilon} \in \operatorname{End}_{\mathfrak{f}}(\mathfrak{Q})$ by $E^{\delta}=E+R_{\overline{E 1}}$ and $E^{\varepsilon}=E-$ $L_{E 1+\overline{E 1}}$. It is easy to check using (1.2) that

$$
V_{x, y}^{\varepsilon}=-V_{y, x} \text { and } V_{x, y}^{\delta} s=-\psi(x, s y)
$$

for $x, y \in \mathbb{Q}, s \in \mathcal{S}$. Let $\operatorname{Strl}\left(\mathscr{Q},,^{-}\right)$be the set of all $E \in \operatorname{End}_{\mathfrak{f}}(\mathscr{Q})$ such that $\left[E, V_{x, y}\right]=V_{E x, y}+V_{x, E^{t} y}$ for all $x, y \in \mathbb{Q}$. Then, $\operatorname{Str}\left(\mathbb{Q}^{-}\right)$is a subalgebra of the Lie algebra $\operatorname{End}_{\mathfrak{f}}(\mathscr{Q})^{-}$containing $\left\{V_{x, y} \mid x, y \in \mathscr{Q}\right\}$, and the map $E \rightarrow E^{\varepsilon}$ is a Lie algebra automorphism of period 2 of $\operatorname{Strl}\left(Q,^{-}\right)$[1, Corollary 5]. Moreover, if $E \in \operatorname{Strl}\left(\mathscr{Q},{ }^{-}\right)$, then $E$ stabilizes $\mathcal{S} \mathscr{Q}$,

$$
E(s x)=E^{\delta}(s) x+s E^{\varepsilon}(x),
$$


$E^{\delta}$ stabilizes $\mathcal{S}$, and

$$
E^{\delta} \psi(x, y)=\psi(E x, y)+\psi(x, E y)
$$

for $s \in \mathcal{S}, x, y \in \mathbb{Q}$ (see [1, $\$ 1]$ and [2, Lemma 1]).

If $u \in \mathbb{Q}, u$ is said to be conjugate invertible if there exists $\hat{u} \in \mathbb{Q}$ such that $V_{\hat{u}, u}=I d$ (or equivalently $V_{u, \hat{u}}=I d$ (by $(1.9)$ and $I d^{\varepsilon}=-I d$ )). If every nonzero element of $\mathscr{Q}$ is conjugate invertible, we say that $\left(Q,,^{-}\right)$is a conjugate division algebra. When no ambiguity exists we often omit the word conjugate in the above terms. If $u$ is invertible, then the element $\hat{u}$ is uniquely determined and is called the conjugate inverse of $u$. Indeed if $u$ is invertible, $U_{u}$ is invertible and $\hat{u}=U_{u}^{-1} u$ (see $[3, \S 6]$ ). If $s \in \mathcal{S}$, then $s$ is invertible if and only if $L_{s}$ is invertible, in which case $\hat{s}=-L_{s}^{-1} 1$, $\hat{s} \in \mathcal{S}$, and $\hat{s} s=s \hat{s}=-1[3, \S 11]$.

Suppose $u \in \mathbb{Q}$ is invertible. The $u$-conjugate isotope $\left(\mathscr{Q},,^{-}\right)^{\langle u\rangle}$ was introduced in [3]. It is a structurable algebra with underlying vector space $\left(Q,{ }^{-}\right)$. The multiplicative identity of $\left(Q,,^{-}\right)^{\langle u\rangle}$ is $1^{\langle u\rangle}=\hat{u}$, the involution $\tau^{\langle u\rangle}$ is given by

$$
\tau^{\langle u\rangle} x=\bar{x}^{\langle u\rangle}=2 x-\{x, u, \hat{u}\}
$$

and the $V$-operators are given by

$$
V_{x, y}^{\langle u\rangle} z=\{x, y, z\}^{\langle u\rangle}=\left\{x, P_{u} y, z\right\},
$$

where

$$
P_{u}=U_{u}\left(2 \tau^{\langle u\rangle}-I d\right)^{-1}
$$

Moreover, the operator $P_{u}$ is invertible with $P_{u}^{-1}=P_{\hat{u}}$ and $P_{u} \hat{u}=u$. (See $[3, \S 7]$ for these facts.) Also if $x \in \mathbb{Q}$, we have $\tau^{\langle u\rangle} x=2 x-\{\hat{u}, u, x\}-\psi(x, \hat{u}) u$ (by (1.3)). Thus,

$$
\tau^{\langle u\rangle} x=x-\psi(x, \hat{u}) u
$$

for $x \in \mathbb{Q}$. Also if $w$ is invertible, then $P_{u} w$ is invertible and $\left(\left(\mathbb{Q},{ }^{-}\right)^{\langle u\rangle}\right)^{\langle w\rangle}=$ $\left(\mathbb{Q},{ }^{-}\right)^{\left\langle P_{u^{w}}\right\rangle}$ (see [3, Proposition 7.2]). Hence if $w=P_{\hat{u}} 1,\left(\left(\mathbb{Q},{ }^{-}\right)^{\langle u\rangle}\right)^{\left\langle u^{*}\right\rangle}=\left(\mathbb{Q},,^{-}\right)^{\langle 1\rangle}=$ $\left(Q,,^{-}\right)$and so $\left(Q,,^{-}\right)$is a conjugate isotope of $\left(Q,,^{-}\right)^{\langle u\rangle}$.

If $u \in \mathbb{Q}$ is invertible, we write $\mathcal{S}^{\langle u\rangle}=\mathcal{S}\left(\left(\mathbb{Q},{ }^{-}\right)^{\langle u\rangle}\right), \mathcal{H}^{\langle u\rangle}=\mathcal{H}\left(\left(\mathbb{Q},{ }^{-}\right)^{\langle u\rangle}\right), \psi^{\langle u\rangle}=$ $\psi_{\left(\Theta,{ }^{-}\right)^{\langle u\rangle}}$, and let $L_{x}^{\langle u\rangle}$ and $R_{x}^{\langle u\rangle}$ denote, respectively, the left and right multiplication operators by $x$ in $\left(Q,{ }^{-}\right)^{\langle u\rangle}$.

Lemma 1.16. Suppose $u \in \mathbb{Q}$ is invertible. Then $\mathcal{S}^{\langle u\rangle}=\mathcal{S} u$. Moreover, if $x, y \in \mathbb{Q}$, $s \in \delta$,

$$
\begin{gathered}
\psi^{\langle u\rangle}(x, y)=\psi(x, y) u, \\
L_{s u}^{\langle u\rangle}=L_{s} P_{u},
\end{gathered}
$$

and

$$
P_{u}(s u)=-\frac{1}{3} U_{u}(s u)
$$


Proof. It follows from (1.15) that $\delta^{\langle u\rangle} \subseteq \delta u$. On the other hand if $s \in \delta$, $\tau^{\langle u\rangle}(s u)=s u+\psi(\hat{u}, s u) u($ by $(1.15))=s u-V_{\hat{u}, u}^{\delta} s($ by $(1.9))=s u-2 s u\left(\right.$ since $I d^{\delta}$ $=2 I d)=-s u$. Thus, $\varsigma^{\langle u\rangle}=\S u$. Next

$$
\begin{gathered}
\left.\psi^{\langle u\rangle}(x, y)=\left\{x, 1^{\langle u\rangle}, y\right\}^{\langle u\rangle}-\left\{y, 1^{\langle u\rangle}, x\right\}^{\langle u\rangle} \quad \text { (by }(1.3)\right) \\
\left.=\left\{x, P_{u} \hat{u}, y\right\}-\left\{y, P_{u} \hat{u}, x\right\}=\{x, u, y\}-\{y, u, x\} \quad \text { (since } P_{u} \hat{u}=u\right) \\
=\psi(x, y) u \quad(\text { by }(1.3)) .
\end{gathered}
$$

Also, in $\left(\mathscr{Q},{ }^{-}\right), 2 s x=\psi(s, 1) x=\left(U_{s, 1}-U_{1, s}\right) x$. Thus in $\left(\mathscr{Q},{ }^{-}\right)^{\langle u\rangle}$,

$$
\begin{aligned}
2 L_{s u}^{\langle u\rangle} x & =\left\{s u, x, 1^{\langle u\rangle}\right\}^{\langle u\rangle}-\left\{1^{\langle u\rangle}, x, s u\right\}^{\langle u\rangle} \\
& =\left\{s u, P_{u} x, \hat{u}\right\}-\left\{\hat{u}, P_{u} x, s u\right\} \\
& =-\psi(\hat{u}, s u) P_{u} x \quad(\text { by }(1.3)) \\
& =\left(V_{\hat{u}, u}^{\delta} s\right) P_{u} x \quad(\text { by }(1.9)) \\
& =2 s P_{u} x .
\end{aligned}
$$

This proves (1.18), (1.19) follows immediately from (1.14) since $s u \in \mathcal{S}^{\langle u\rangle}$.

The multiplication algebra of $\left(Q,,^{-}\right)$is the associative subalgebra of $\operatorname{End}_{\mathrm{f}}(\mathbb{Q})$ generated by $\tau,\left\{L_{x} \mid x \in \mathbb{Q}\right\}$ and $\left\{R_{x} \mid x \in \mathbb{Q}\right\}$, where $\tau$ denotes the involution of $\left(\mathscr{Q},{ }^{-}\right)$[10, p. 207]. Since $R_{x}=\tau L_{\vec{x}} \tau$, this algebra is generated by $\tau$ and $\left\{L_{x} \mid x \in \mathbb{Q}\right\}$. But $L_{h}=V_{h, 1}$ for $h \in \mathcal{H}$ and $L_{s}=-\frac{1}{3} V_{s, 1}-\frac{2}{3} \tau V_{s, 1} \tau$ for $s \in \mathcal{S}$. Thus, the multiplication algebra of $\left(\mathbb{Q},{ }^{-}\right)$is generated by $\left\{V_{x, y} \mid x, y \in \mathbb{Q}\right\}$ and $\tau$. The center of $\left(\mathbb{Q},^{-}\right)$is the set $\mathscr{Z}\left(\mathbb{Q},,^{-}\right)$of all elements of the center of $Q$ that are fixed by ${ }^{-}$. Then, $\mathscr{Z}\left(\mathbb{Q},,^{-}\right)$can be identified with the centralizer in $\operatorname{End}_{f}(Q)$ of the multiplication algebra of $\left(Q,,^{-}\right)[10$, pp. 207-208].

By an ideal of $\left(\mathscr{Q},{ }^{-}\right)$, we mean an ideal of $Q$ that is stabilized by ${ }^{-}$. We say that $\left(Q,,^{-}\right)$is simple if the only ideals of $\left(Q,,^{-}\right)$are $\{0\}$ and $Q$. We say that $\left(Q,,^{-}\right)$is central simple if $\left(\mathbb{Q},{ }^{-}\right)$is simple and $\mathscr{Z}\left(\mathbb{Q},,^{-}\right)=k 1$.

Lemma 1.20. Suppose $u \in Q$ is invertible. Then, $\left(\mathbb{Q},{ }^{-}\right)$and $\left(Q,,^{-}\right)^{\langle u\rangle}$ have the same multiplication algebras. Hence, $\left(Q,^{-}\right)$is simple (resp. central simple) if and only if $\left(Q,,^{-}\right)^{\langle u\rangle}$ is simple (resp. central simple).

Proof. From (1.12) and (1.13) it follows that the multiplication algebra of $\left(Q,,^{-}\right)^{\langle u\rangle}$ is contained in the multiplication algebra of $\left(Q,,^{-}\right)$. Since $\left(Q,,^{-}\right)$is an isotope of $\left(Q,,^{-}\right)^{\langle u\rangle}$, the reverse inclusion follows.

Suppose $\left(\mathbb{Q}^{\prime},-\right)$ is another structurable algebra. An isotopy of $(\mathbb{Q},-)$ onto $\left(\mathbb{Q}^{\prime},-\right)$ is an invertible $\mathfrak{f}$-linear map $\alpha: \mathbb{Q} \rightarrow \mathbb{Q}^{\prime}$ such that

$$
\alpha\{x, y, z\}=\{\alpha x, \hat{\alpha} y, \alpha z\}
$$

for some $\mathfrak{f}$-linear $\hat{\alpha}: \mathbb{Q} \rightarrow \mathbb{Q}^{\prime}$ and all $x, y, z \in \mathbb{Q}$. In that case, $\hat{\alpha}$ is uniquely determined and there exists a unique $f^{f}$-linear bijection $\alpha_{\S}: \delta \rightarrow \delta^{\prime}$ such that

$$
\alpha_{\S} \psi(x, y)=\psi^{\prime}(\alpha x, \alpha y) \text { and } \alpha(s x)=\alpha_{\S}(s) \hat{\alpha}(x)
$$


for $x, y \in \mathbb{Q}, s \in \mathcal{S}$, where $\mathcal{S}^{\prime}=S\left(\mathcal{Q}^{\prime},,^{-}\right)$and $\psi^{\prime}=\psi_{\left(\mathbb{Q}^{\prime},-\right)}[3, \S \S 8$ and 12]. If there exists an isotopy from $\left(\mathbb{Q},,^{-}\right)$onto $\left(\mathbb{Q}^{\prime},,^{-}\right)$, we say that $\left(\mathcal{Q},,^{-}\right)$and $\left(\mathbb{Q}^{\prime},,^{-}\right)$are isotopic. $\left(\mathscr{Q},,^{-}\right)$and $\left(\mathscr{Q}^{\prime},-\right)$ are isotopic if and only if $\left(\mathscr{Q}^{\prime},-\right)$ is isomorphic to $\left(\mathscr{Q},,^{-}\right)^{\langle u\rangle}$ for some invertible $u \in \mathbb{Q}[3$, Proposition 8.5].

Denote by $\Gamma\left(\mathcal{Q},{ }^{-}\right)$the group of all isotopies of $\left(\Theta,,^{-}\right)$onto itself. If $s \in \delta$ and $u \in \mathbb{U}^{\prime}$ are invertible, then $L_{s} \in \Gamma\left(\mathbb{Q},{ }^{-}\right), P_{u} \in \Gamma\left(\mathbb{U},{ }^{-}\right)$,

$$
\hat{L}_{s}=L_{\hat{s}}=-L_{s}^{-1} \text { and } \hat{P}_{u}=P_{\hat{u}}=P_{u}^{-1}
$$

[3, Proposition 11.1 and Theorem 8.3].

2. Simple structurable algebras with $\operatorname{dim}_{\mathrm{f}}(\S)=1$. Structurable algebras $\left(\leftrightarrow,{ }^{-}\right)$ with $\operatorname{dim}_{\mathrm{f}} \delta\left(\Theta,,^{-}\right)=0$ are just Jordan algebras (see $\left.[\mathbf{1}, \S 1]\right)$. We are interested in this paper in the next case when $\operatorname{dim}_{\mathrm{f}} \mathcal{S}\left(\mathbb{Q},,^{-}\right)=1$. We assume in this section that $\left(\mathbb{Q},{ }^{-}\right)$ is a simple structurable algebra over $\mathfrak{f}$ and that $\operatorname{dim}_{\mathrm{f}}(\delta)=1$, where $S=S\left(\mathscr{Q},{ }^{-}\right)$. We

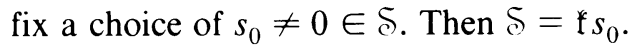

LEMMA 2.1. (a) $s_{0}$ is invertible.

(b) There exists $\mu \neq 0 \in \mathfrak{f}$ such that $\hat{s}_{0}=-\mu^{-1} s_{0}, s_{0}^{2}=\mu 1$, and $L_{s_{0}}^{2}=R_{s_{0}}^{2}=\mu I d$.

(c) $\left(\mathbb{Q},{ }^{-}\right)$is central simple.

Proof. (a) By (1.10), $S \mathbb{Q}$ is stabilized by $\operatorname{Strl}\left(\mathbb{Q},{ }^{-}\right)$. Also if $s \in \mathcal{S}, x \in \mathbb{Q}$, $\overline{s x}=s x+\overline{s x}-s x$ and so $\overline{\delta Q} \subseteq \delta Q+\delta \subseteq \delta Q$. Hence, $\delta Q$ is stabilized by ${ }^{-}$. Thus, $S \mathscr{Q}$ is stabilized by the multiplication algebra of $\left(\mathscr{Q},{ }^{-}\right)$. Therefore, since $\left(\mathscr{Q},,^{-}\right)$is simple, $\delta \mathbb{Q}=\mathbb{Q}$ and so $s_{0} \mathbb{Q}=\mathbb{Q}$. Thus, $1=s_{0} t$ for some $t \in \mathbb{Q}$. Now $U_{1} x=2 \bar{x}-x$ for $x \in Q$ and so $U_{1}$ is invertible. But $U_{1}=U_{1,1}=U_{s_{0} t, s_{0} t}=-L_{s_{0}} U_{t} L_{s_{0}}$ (by (1.6)). Thus $L_{s_{0}}$ is invertible and so $s_{0}$ is invertible.

(b) Since $\hat{s}_{0} \in \mathfrak{S}, \hat{s}_{0}=-\mu^{-1} s_{0}$ for some $\mu \neq 0 \in \mathfrak{f}$. Since $s_{0} \hat{s}_{0}=-1$, we have $s_{0}^{2}=\mu 1$. The last equations then follow using (1.2).

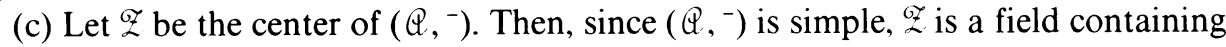
f1. Moreover, $1=\operatorname{dim}_{\mathfrak{f}} \delta=\left(\operatorname{dim}_{\mathscr{Q}} \delta\right)\left(\operatorname{dim}_{\mathrm{f}} \mathscr{Z}\right)$ and so $\operatorname{dim}_{\mathrm{f}} \mathscr{Z}=1$. Thus, $\mathscr{Z}=\mathfrak{f} 1$.

LEMMA 2.2. $\psi$ is a nondegenerate skew-symmetric bilinear mapping from $\leftrightarrow \times \notin$ to $\mathcal{S}$.

Proof. By (1.11) the radical $\Re$ of $\psi$ is stabilized by $\operatorname{Strl}\left(Q,{ }^{-}\right)$. Also if $x \in \mathcal{R}$, we have $0=\psi(x, 1)=x-\bar{x}$ and so $x \in \mathcal{H}$. Thus, $\mathscr{R} \subseteq \mathcal{H}$ and so $\overline{\mathcal{R}} \subseteq \mathcal{R}$. Therefore, $\mathcal{R}$ is an ideal of $\left(\Theta,{ }^{-}\right)$. But $\psi\left(s_{0}, 1\right)=2 s_{0} \neq 0$ and so $1 \notin \Re$. Thus, since $\left(\mathscr{Q},,^{-}\right)$is simple, $\mathscr{R}=\{0\}$.

Define $\chi_{(Q,-)}: \mathscr{Q} \times \mathscr{Q} \rightarrow \mathfrak{f}$ by

$$
\chi_{(\Theta,-)}(x, y)=\frac{2}{\mu} \psi_{\left(Q_{,-}^{-}\right)}\left(s_{0} x, y\right) s_{0}=\frac{2}{\mu}\left(V_{y, x}^{\delta} s_{0}\right) s_{0},
$$

where here we are identifying $\mathfrak{f}$ with $\mathfrak{l}$ and using (1.9). If no ambiguity exists, we write $\chi=\chi_{\left(\mathbb{Q}^{-}\right)}$. Then, $\chi(1,1)=4$ and $\chi$ is independent of the choice of $s_{0}$. Finally, using Lemma 2.1(b), we have

$$
\psi(x, y)=\frac{1}{2 \mu} \chi\left(s_{0} x, y\right) s_{0} \quad \text { and } \quad \psi(x, y)\left(s_{0} z\right)=\frac{1}{2} \chi\left(s_{0} x, y\right) z
$$

for $x, y, z \in \mathbb{Q}$. 
Proposition 2.5. $\chi$ is a nondegenerate symmetric bilinear form such that

$$
\begin{gathered}
\chi(E x, y)=-\chi\left(x, E^{\varepsilon} y\right), \\
\chi(\bar{x}, \bar{y})=\chi(x, y), \\
\chi(x y, z)=\chi(y, \bar{x} z)=\chi(x, z \bar{y}), \\
\chi\left(s_{0} x, y\right)=-\chi\left(s_{0} y, x\right), \\
\chi(\{x, y, z\}, w)=\chi(z,\{y, x, w\})=\chi(y,\{z, w, x\})=\chi(x,\{w, z, y\})
\end{gathered}
$$

for $x, y, z, w \in Q$ and $E \in \operatorname{Strl}\left(Q,,^{-}\right)$.

Proof. First of all,

$$
\begin{aligned}
\psi\left(s_{0} x, y\right) & =\frac{1}{\mu} \psi\left(s_{0} x, s_{0}\left(s_{0} y\right)\right)=-\frac{1}{\mu} s_{0} \psi\left(x, s_{0} y\right) s_{0} \\
& =-\psi\left(x, s_{0} y\right),
\end{aligned}
$$

since $\mathfrak{S}=\mathfrak{f} s_{0}$. Thus, $\chi$ is symmetric. $\chi$ is nondegenerate by Lemmas 2.1 and 2.2 . Suppose next that $E \in \operatorname{Strl}\left(\mathbb{Q},{ }^{-}\right)$. Then, $\psi\left(s_{0} x, E y\right)=E^{\delta} \psi\left(s_{0} x, y\right)-\psi\left(E\left(s_{0} x\right), y\right)$ (by $(1.11))=E^{\delta} \psi\left(s_{0} x, y\right)-\psi\left(E^{\delta}\left(s_{0}\right) x, y\right)-\psi\left(s_{0} E^{\varepsilon} x, y\right)$ (by (1.10)). Since $\delta$ is 1-dimensional, $E^{\delta} \delta \mid \lambda I d$ for some $\lambda \in \mathfrak{f}$. Therefore, $\psi\left(s_{0} x, E y\right)=-\psi\left(s_{0} E^{\varepsilon} x, y\right)$. Replacing $E$ by $E^{\varepsilon}$, this gives the first equation. To prove (2.6), it suffices to show that $\chi\left(s_{0}, h\right)=0$ for $h \in \mathcal{H}\left(Q,{ }^{-}\right)$. But $\chi\left(s_{0}, h\right)=2 \psi\left(s_{0}^{2}, h\right) \mu^{-1} s_{0}=2 \psi(1, h) s_{0}=0$ and so we have (2.6). In view of (2.6), we need only prove the first equation of (2.7). But if $x=h \in \mathcal{H}\left(\mathbb{Q},{ }^{-}\right), \chi(h y, z)=\chi\left(V_{h, 1} y, z\right)=-\chi\left(y, V_{h, 1}^{\varepsilon} z\right)=\chi\left(y, V_{1, h} z\right)=$ $\chi(y, h z)$. On the other hand if $x=s_{0}, \chi\left(s_{0} y, z\right)=-\chi\left(s_{0} z, y\right)$ (by $\left.(2.4)\right)=$ $-\chi\left(y, s_{0} z\right)$ and so we have (2.7) and (2.8). The equations in (2.9) follow immediately using (2.6) and (2.7).

Define a quartic form $\nu_{(\mathscr{Q},-)}: \mathbb{Q} \rightarrow \mathfrak{f}$ by

$$
\nu_{(\mathbb{Q},-)}(x)=\frac{1}{12 \mu} \chi_{(\mathbb{Q},-)}\left(s_{0} x,\left\{x, s_{0} x, x\right\}\right) .
$$

Again if no ambiguity exists, we write $\nu=\nu_{\left(Q_{-}\right)}$. It is easy to check that $\nu(1)=1$ and that $\nu$ is independent of the choice of $s_{0}$.

Proposition 2.11. Suppose $x \in \mathbb{Q}$. Then,

$$
V_{x, s_{0}\left\{x, s_{0} x, x\right\}}=-3 \mu \nu(x) I d .
$$

Moreover, $x$ is invertible if and only if $\nu(x) \neq 0$, in which case

$$
\hat{x}=-\frac{1}{3 \mu \nu(x)} s_{0}\left\{x, s_{0} x, x\right\} \text {. }
$$

Proof.

$$
\begin{aligned}
V_{x, s_{0}\left\{x, s_{0} x, x\right\}} & =V_{\left\{x, s_{0} x, x\right\}, s_{0} x}-L_{\psi\left(x,\left\{x, s_{0} x, x\right\}\right)} L_{s_{0}} \\
& =-\frac{1}{2} L_{\psi\left(x,\left\{x, s_{0} x, x\right\}\right)} L_{s_{0}} \quad(\text { by }(1.8)) \\
& =-\frac{1}{4} \chi\left(s_{0} x,\left\{x, s_{0} x, x\right\}\right) I d \quad \text { (by } \\
& =-3 \mu \nu(x) I d
\end{aligned}
$$


and so we have (2.12). If $\nu(x) \neq 0,(2.12)$ implies that $x$ is invertible and (2.13) holds. Finally, suppose that $\nu(x)=0$. Then, by (2.12), $V_{x, s_{0}\left\{x, s_{0} x, x\right\}}=0$. Applying both sides to $x$ gives $U_{x} L_{s_{0}} U_{x} L_{s_{0}} x=0$. Thus, $U_{x}$ is not invertible and so $x$ is not invertible.

Define $\nu(x, y, z, w)=\left.\partial_{y} \partial_{z} \partial_{w} \nu\right|_{x}$ for $x, y, z, w \in \mathcal{Q}$. Then $\nu(,,$,$) is the unique$ symmetric 4-linear form on $\mathbb{Q}$ such that

$$
\nu(x, x, x, x)=24 \nu(x) .
$$

Proposition 2.14. If $x, y, z, w \in \mathbb{Q}$, then

$$
\begin{aligned}
\nu(x, y, z, w)= & \frac{2}{\mu} \chi\left(s_{0} x,\left\{y, s_{0} z, w\right\}\right)-\frac{1}{2 \mu} \chi\left(s_{0} x, y\right) \chi\left(s_{0} z, w\right) \\
& -\frac{1}{2 \mu} \chi\left(s_{0} x, w\right) \chi\left(s_{0} z, y\right)-\frac{1}{2 \mu} \chi\left(s_{0} x, z\right) \chi\left(s_{0} y, w\right) .
\end{aligned}
$$

Proof. Temporarily denote the right-hand side of $(2.15)$ by $R(x, y, z, w)$. Since $\chi\left(s_{0} x, x\right)=0$ (by $(2.8)$ ), $R(x, x, x, x)=24 \nu(x)$. Hence it suffices to show that $R(x, y, z, w)$ is symmetric in its arguments. Now $\chi\left(s_{0} x,\left\{y, s_{0} z, w\right\}\right)=$ $\chi\left(s_{0} z,\left\{w, s_{0} x, y\right\}\right)$ by (2.9) and hence, using (2.8), it follows that $R(x, y, z, w)=$ $R(z, w, x, y)$. Thus, since the permutations (13)(24), (24), and (23) generate $S_{4}$, it suffices to show that $R(x, y, z, w)=R(x, w, z, y)=R(x, z, y, w)$. Now

$$
\begin{aligned}
\chi\left(s_{0} x,\left\{y, s_{0} z, w\right\}\right) & \left.=\chi\left(s_{0} x,\left\{w, s_{0} z, y\right\}+\psi(y, w)\left(s_{0} z\right)\right) \quad \text { (by }(1.3)\right) \\
& =\chi\left(s_{0} x,\left\{w, s_{0} z, y\right\}\right)+\frac{1}{2} \chi\left(s_{0} x, z\right) \chi\left(s_{0} y, w\right)
\end{aligned}
$$

and so we have $R(x, y, z, w)=R(x, w, z, y)$. Finally

$$
\begin{aligned}
\chi\left(s_{0} x,\left\{y, s_{0} z, w\right\}\right) & =\chi\left(s_{0} x,\left\{z, s_{0} y, w\right\}-\psi(y, z)\left(s_{0} w\right)\right) \quad(\text { by } \\
& =\chi\left(s_{0} x,\left\{z, s_{0} y, w\right\}\right)-\frac{1}{2} \chi\left(s_{0} x, w\right) \chi\left(s_{0} y, z\right)
\end{aligned}
$$

and we have $R(x, y, z, w)=R(x, z, y, w)$.

Define

$$
\langle x, y\rangle_{F}=\psi(x, y) s_{0}=\frac{1}{2} \chi\left(s_{0} x, y\right)
$$

and

$$
(y, z, w)_{F}=2\left\{y, s_{0} z, w\right\}-\langle z, w\rangle_{F} y-\langle z, y\rangle_{F} w-\langle y, w\rangle_{F} z
$$

for $x, y, z, w \in \mathbb{Q}$. Then, $\langle,\rangle_{F}$ is a nondegenerate skew-symmetric form on $\mathbb{Q}$,

$$
(x, x, x)_{F}=2\left\{s, s_{0} x, x\right\} \quad \text { and } \quad\left\langle x,(y, z, w)_{F}\right\rangle_{F}=\frac{\mu}{2} \nu(x, y, z, w)
$$

(by $(2.15))$.

Proposition 2.18. The product $(,,)_{F}$ and the skew-symmetric form $\langle,\rangle_{F}$ give $\mathbb{Q}$ the structure of a simple Freudenthal triple system.

Proof. In order to show that $\mathbb{Q}$ is a Freudenthal triple system, we must show by definition that the product $(x, y, z)_{F}$ and the 4-linear form $\left\langle x,(y, z, w)_{F}\right\rangle_{F}$ are symmetric in their arguments and that

$$
\left(x,(x, x, x)_{F}, y\right)_{F}=\langle y, x\rangle_{F}(x, x, x)_{F}+\left\langle y,(x, x, x)_{F}\right\rangle_{F} x
$$


for $x, y \in \mathbb{Q}$ [7]. The first two conclusions follow from (2.17), while (2.19) follows immediately from (2.12) and (2.17). Finally, according to Theorem 1 of [7], simplicity follows from the nondegeneracy of $\langle,\rangle_{F}$.

Thus $(x, y, z)_{F}$ is the symmetric ternary product that results from polarizing $2\left\{x, s_{0} x, x\right\}$.

3. Isotopes. Suppose once again that $\left(Q,,^{-}\right)$is a simple structurable algebra over $\mathfrak{f}$ with $\delta=\mathfrak{f} s_{0}, s_{0} \neq 0 \in \mathbb{Q}$. Let $u$ be an invertible element of $Q$. Then by (1.15) and (2.4) the involution $\tau^{\langle u\rangle}$ on $\left(\mathscr{Q},{ }^{-}\right)^{\langle u\rangle}$ is given by

$$
\tau^{\langle u\rangle} x=x-\frac{1}{2 \mu} \chi\left(s_{0} x, \hat{u}\right) s_{0} u
$$

for $x \in \mathbb{Q}$. Moreover, by Lemma 1.16.

$$
\delta^{\langle u\rangle}=\mathfrak{f} s_{0}^{\langle u\rangle}
$$

where $s_{0}^{\langle u\rangle}=s_{0} u \neq 0$. Hence, by Lemma 1.20, $\left(\mathbb{Q},^{-}\right)^{\langle u\rangle}$ is a simple structurable algebra with $\operatorname{dim}_{f} \delta^{\langle u\rangle}=1$. We write $\chi^{\langle u\rangle}=\chi_{(\Theta,-)^{\langle u\rangle}}$ and $\nu^{\langle u\rangle} \nu_{(\Theta,-)^{\prime}}$. We wish to compute these forms.

LEMMA 3.2. The square of $s_{0}^{\langle u\rangle}$ in $\left(Q,^{-}\right)^{\langle u\rangle}$ is $\mu^{\langle u\rangle} 1^{\langle u\rangle}$, where $\mu^{\langle u\rangle}=\nu(u) \mu$.

Proof. In $\left(\mathbb{Q},{ }^{-}\right), s_{0}^{2}=\frac{1}{3}\left\{s_{0}, 1, s_{0}\right\}$. Hence, the required square is $\frac{1}{3}\left\{s_{0}^{\langle u\rangle}, 1^{\langle u\rangle}, s_{0}^{\langle u\rangle}\right\}^{\langle u\rangle}=\frac{1}{3}\left\{s_{0} u, u, s_{0} u\right\} \quad$ (by $\left.(1.13)\right)=-\frac{1}{3} s_{0}\left\{u, s_{0} u, u\right\} \quad$ (by $\left.(1.6)\right)=$ $\mu \nu(u) \hat{u}$ (by (2.13)).

LEMMA 3.3. $\left(P_{u}\right)_{\S}\left(s_{0}\right)=\nu(u) s_{0}$ (using the notation of (1.22) with $\alpha=P_{u}$ ),

$$
P_{u} L_{s_{0}} P_{u}=\nu(u) L_{s_{0}} \text { and } L_{s_{0}} P_{u} L_{s_{0}}=\mu \nu(u) P_{\hat{u}} .
$$

PROOF. First of all $\left(P_{u}\right)_{\S}\left(s_{0}\right)=\rho s_{0}$ for some $\rho \neq 0 \in \mathfrak{f}$. Then, $P_{u}\left(s_{0} u\right)=\left(\rho s_{0}\right) \hat{P}_{u} u$ (by $(1.22))=\rho s_{0} P_{\hat{u}} u \quad($ by $(1.23))=\rho s_{0} \hat{u}=-(3 \nu(u))^{-1} \rho U_{u}\left(s_{0} u\right)$ (by (2.13). But $P_{u}\left(s_{0} u\right)=-\frac{1}{3} U_{u}\left(s_{0} u\right)$ (by (1.19)) and so $\rho=\nu(u)$. But then by (1.22), $P_{u} L_{s_{0}}=$ $\nu(u) L_{s_{0}} \hat{P}_{u}$ which together with (1.23) implies (3.4).

Proposition 3.5. If $x, y, u \in \mathbb{Q}$ and $u$ is invertible, then

$$
\chi^{\langle u\rangle}(x, y)=\chi\left(P_{u} x, y\right)=\chi\left(x, P_{u} y\right)
$$

and

$$
\nu^{\langle u\rangle}(x)=\nu(u) \nu(x) .
$$

Proof. By (2.3), $\chi(x, y) s_{0}=2 \psi\left(s_{0} x, y\right)$. The corresponding equation in $\left(\mathscr{Q},{ }^{-}\right)^{\langle u\rangle}$ gives $\chi^{\langle u\rangle}(x, y) s_{0} u=2 \psi^{\langle u\rangle}\left(L_{s_{0} u}^{\langle u\rangle}, y\right)=2 \psi\left(s_{0} P_{u} x, y\right) u$ (by (1.17) and (1.18)) $=$ $\chi\left(P_{u} x, y\right) s_{0} u$ (by (2.4)), which proves (3.6) since $\chi^{\langle u\rangle}$ and $\chi$ are symmetric. Next by (2.10) applied to $\left(Q,^{-}\right)^{\langle u\rangle}$, we have

$$
\begin{array}{rlr}
12 \mu^{\langle u\rangle} \nu^{\langle u\rangle}(x) & =\chi^{\langle u\rangle}\left(L_{s_{0} u}^{\langle u\rangle} x,\left\{x, L_{s_{0} u}^{\langle u\rangle}, x\right\}^{\langle u\rangle}\right)=\chi\left(P_{u} L_{s_{0}} P_{u} x,\left\{x, P_{u} L_{s_{0}} P_{u} x, x\right\}\right) \\
& =\nu(u)^{2} \chi\left(s_{0} x,\left\{x, s_{0} x, x\right\}\right) & (\text { by }(3.6),(1.18) \text { and (1.13)) } \\
& \left.\left.=12 \mu \nu(u)^{2} \nu(x) .4\right)\right)
\end{array}
$$


Since $\mu^{\langle u\rangle}=\nu(u) \mu$, this implies (3.7).

$\nu$ admits composition in the following sense:

PROPOSITION 3.8. If $u, x \in \mathbb{P}$ and $u$ is invertible, then

$$
\nu\left(P_{u} x\right)=\nu(u)^{2} \nu(x)
$$

and

$$
\nu\left(s_{0} x\right)=\mu^{2} \nu(x) .
$$

PROOF.

$$
\begin{aligned}
12 \mu \nu\left(P_{u} x\right) & =\chi\left(s_{0} P_{u} x,\left\{P_{u} x, s_{0} P_{u} x, P_{u} x\right\}\right) \\
& =\chi\left(s_{0} P_{u} x, P_{u}\left\{x, P_{u} s_{0} P_{u} x, x\right\}\right) \\
& =\chi\left(P_{u} s_{0} P_{u} x,\left\{x, P_{u} s_{0} P_{u} x, x\right\}\right) \\
& =\nu(u)^{2} \chi\left(s_{0} x,\left\{x, s_{0} x, x\right\}\right) \\
& =12 \mu \nu(u)^{2} \nu(x) .
\end{aligned}
$$

Also

$$
\begin{aligned}
12 \mu \nu\left(s_{0} x\right) & =\chi\left(s_{0}\left(s_{0} x\right),\left\{s_{0} x, s_{0}\left(s_{0} x\right), s_{0} x\right\}\right)=\mu^{2} \chi\left(x,\left\{s_{0} x, x, s_{0} x\right\}\right) \\
& =\mu^{2} \chi\left(s_{0} x,\left\{x, s_{0} x, x\right\}\right) \quad \text { (by (1.6) and (2.8)) } \\
& =12 \mu^{3} \nu(x), \quad \square
\end{aligned}
$$

4. Matrix algebras. Let $\mathcal{G}$ and $\mathcal{G}^{\prime}$ be vector spaces over ${ }^{*}$ possessing cubic forms $N$ and $N^{\prime}$, respectively, and paired by a nondegenerate bilinear form $T: \mathscr{G} \times \mathscr{q}^{\prime} \rightarrow \mathfrak{f}$. We say that the triple $\left(T, N, N^{\prime}\right)$ is defined on $\left(\mathscr{G}, \mathscr{q}^{\prime}\right)$. If $j \in \mathscr{q}$ and $j^{\prime} \in \mathcal{G}^{\prime}$, let $j^{\#} \in \mathcal{G}^{\prime}$ and $j^{\prime \#^{\prime}} \in \mathcal{y}$ be the elements satisfying

$$
\left.\partial_{k} N\right|_{j}=T\left(k, j^{\#}\right) \quad \text { and }\left.\quad \partial_{k^{\prime}} N^{\prime}\right|_{j^{\prime}}=T\left(j^{\prime \#^{\prime}}, k^{\prime}\right)
$$

respectively, for all $k \in \mathcal{G}$ and $k^{\prime} \in \mathscr{G}^{\prime}$. We say that the triple $\left(T, N, N^{\prime}\right)$ satisfies the adjoint identities if

$$
\left(j^{\#}\right)^{\#^{\prime}}=N(j) j \quad \text { and } \quad\left(j^{\prime \#^{\prime}}\right)^{\#}=N^{\prime}\left(j^{\prime}\right) j^{\prime}
$$

for $j \in \mathscr{g}$ and $j^{\prime} \in g^{\prime}$. Of course if $N=0$ and $N^{\prime}=0$ these identities are trivially satisfied. If $\left(T, N, N^{\prime}\right)$ satisfies the adjoint identities and either $N \neq 0$ or $N^{\prime} \neq 0$, then both are nonzero and we say that $\left(T, N, N^{\prime}\right)$ is nontrivial. Such triples were first studied in [18].

If $\left(T, N, N^{\prime}\right)$ is a triple defined on $\left(q, g^{\prime}\right)$, define

$$
N(j, k, l)=\left.\partial_{j} \partial_{k} N\right|_{l}, \quad N^{\prime}\left(j^{\prime}, k^{\prime}, l^{\prime}\right)=\left.\partial_{j^{\prime}} \partial_{k^{\prime}} N^{\prime}\right|_{l^{\prime}}
$$

and

$$
j \times k=(j+k)^{\#}-j^{\#}-k^{\#}, \quad j^{\prime} \times k^{\prime}=\left(j^{\prime}+k^{\prime}\right)^{\#^{\prime}}-j^{\prime \#^{\prime}}-k^{\prime \#^{\prime}}
$$

for $j, k, l \in \mathcal{q}, j^{\prime}, k^{\prime}, l^{\prime} \in \mathcal{g}^{\prime}$. Then these expressions are symmetric and linear in their arguments, $N(j)=\frac{1}{6} N(j, j, j), \quad N^{\prime}\left(j^{\prime}\right)=\frac{1}{6} N^{\prime}\left(j^{\prime}, j^{\prime}, j^{\prime}\right), \quad j^{\#}=\frac{1}{2} j \times j, j^{\prime \#^{\prime}}$ $=\frac{1}{2} j^{\prime} \times j^{\prime}, N(j, k, l)=T(j, k \times l)$ and $N^{\prime}\left(j^{\prime}, k^{\prime}, l^{\prime}\right)=T\left(j^{\prime} x^{\prime} k^{\prime}, l^{\prime}\right)$ for $j, k, l \in \mathcal{G}$, $j^{\prime}, k^{\prime}, l^{\prime} \in q^{\prime}$. 
Suppose $\left(T, N, N^{\prime}\right)$ is a triple satisfying the adjoint identities. We define an algebra with involution $\Re\left(T, N, N^{\prime}\right)$ as follows: The underlying vector space is

$$
\left\{\left[\begin{array}{ll}
\alpha & j \\
j^{\prime} & \beta
\end{array}\right] \mid \alpha, \beta \in \mathfrak{k}, j \in \mathcal{F}, j^{\prime} \in \mathscr{F}^{\prime}\right\} .
$$

The product and involution are given by

$$
\left[\begin{array}{ll}
\alpha & j \\
j^{\prime} & \beta
\end{array}\right]\left[\begin{array}{ll}
\gamma & k \\
k^{\prime} & \delta
\end{array}\right]=\left[\begin{array}{ll}
\alpha \gamma+T\left(j, k^{\prime}\right) & \alpha k+\delta j+j^{\prime} x^{\prime} k^{\prime} \\
\gamma j^{\prime}+\beta k^{\prime}+j \times k & \beta \delta+T\left(k, j^{\prime}\right)
\end{array}\right]
$$

and

$$
\left[\begin{array}{ll}
\alpha & j \\
j^{\prime} & \beta
\end{array}\right]=\left[\begin{array}{ll}
\beta & j \\
j^{\prime} & \alpha
\end{array}\right]
$$

Then, $\Re\left(T, N, N^{\prime}\right)$ is a structurable algebra. Indeed, if $\left(T, N, N^{\prime}\right)$ is non-trivial this has been shown in $[\mathbf{1}, \S 8]$ (where nontrivial triples satisfying the adjoint identities are called admissible triples) and if $\left(T, N, N^{\prime}\right)$ is trivial the same proof works. Also, it is easy to check that $\Re\left(T, N, N^{\prime}\right)$ is simple and that $\delta\left(\Re\left(T, N, N^{\prime}\right)\right)=\mathfrak{f}_{0}$, where $s_{0}=\left[\begin{array}{ll}1 & 0 \\ 0 & -1\end{array}\right]$. Thus, $\Re\left(T, N, N^{\prime}\right)$ is an example of an algebra satisfying the hypotheses of $\S 2$.

Suppose $\left(T, N, N^{\prime}\right)$ is a triple satisfying the adjoint identities. Let $\chi=\chi_{\Re\left(T, N, N^{\prime}\right)}$ and $\nu=\nu_{\mathscr{N}\left(T, N, N^{\prime}\right)}$. It is straightforward to verify that if

$$
x=\left[\begin{array}{cc}
\alpha & j \\
j^{\prime} & \beta
\end{array}\right] \text { and } y=\left[\begin{array}{cc}
\gamma & k \\
k^{\prime} & \delta
\end{array}\right]
$$

then

$$
\chi(x, y)=2 \operatorname{tr}(x \bar{y})=2\left(\alpha \delta+\beta \gamma+T\left(j, k^{\prime}\right)+T\left(k, j^{\prime}\right)\right)
$$

and

$$
\nu(x)=4 \alpha N(j)+4 \beta N^{\prime}\left(j^{\prime}\right)-4 T\left(j^{\prime \#^{\prime}}, j^{\#}\right)+\left(\alpha \beta-T\left(j, j^{\prime}\right)\right)^{2} .
$$

If $\mathcal{g}$ is a separable Jordan algebra over $\mathfrak{k}$ of degree 3 with generic norm $N_{\mathfrak{g}}$ and generic trace $T_{g}$ and $\zeta \neq 0 \in k$, then $\left(\zeta T_{g}, \zeta N_{g}, \zeta^{2} N_{g}\right)$ is a nontrivial triple defined on $(\mathcal{f}, \mathcal{G})$ satisfying the adjoint identities. Moreover, if $\left(T, N, N^{\prime}\right)$ is any nontrivial triple satisfying the adjoint identities and defined on a pair of spaces of dimension bigger than 2 then Springer has shown in $\left[\mathbf{1 7}\right.$ and 18] that $\left(T, N, N^{\prime}\right)$ is isomorphic to $\left(\zeta T_{g}, \zeta N_{g}, \zeta^{2} N_{g}\right)$ for some $\zeta$ and $g$ as above. In that case, $\Re\left(T, N, N^{\prime}\right) \cong$ $\Re\left(\zeta T_{g}, \zeta N_{g}, \zeta^{2} N_{g}\right)$. If $g$ is the 27 -dimensional split exceptional central simple Jordan algebra, then $\Re=\Re\left(T_{g}, N_{g}, N_{g}\right)$ is the algebra referred to in the introduction.

We next obtain characterizations up to isomorphism and isotopy of the matrix algebras $\Re\left(T, N, N^{\prime}\right)$. Earlier proofs of corresponding results for Freudenthal triple systems were given in $[6,7$ and 14$]$.

For the rest of this section assume that $\left(Q,,^{-}\right)$is a simple structurable algebra over $\mathfrak{f}$ and that $\delta=\mathfrak{t} s_{0}, s_{0} \neq 0$. Then $s_{0}^{2}=\mu 1, \mu \in \mathfrak{t}^{*}$. The first result is obtained by adapting part of the proof of Theorem 25 of [1]. 
Proposition 4.5. $\left(Q,,^{-}\right) \cong \mathscr{M}\left(T, N, N^{\prime}\right)$ for some triple $\left(T, N, N^{\prime}\right)$ satisfying the adjoint identities if and only if $\mu \in \mathfrak{t}^{* 2}$.

Proof. The necessity of the condition $\mu \in \mathfrak{l}^{* 2}$ is clear. To prove the sufficiency suppose that $\mu \in \mathfrak{f}^{* 2}$. Replacing $s_{0}$ by a nonzero multiple of $s_{0}$, we may assume that $\mu=1$. Let $\mathcal{E}=\mathfrak{1} \oplus k s_{0}$ and $\mathscr{Q}=\left\{x \in \mathscr{Q} \mid \chi(1, x)=\chi\left(s_{0}, x\right)=0\right\}$. Since $\chi(1,1)=$ 4, $\chi\left(s_{0}, s_{0}\right)=-\chi\left(1, s_{0}^{2}\right)=-\chi(1,1)=-4$, and $\chi\left(1, s_{0}\right)=0$, it follows that $\mathscr{Q}=\mathcal{E} \oplus$

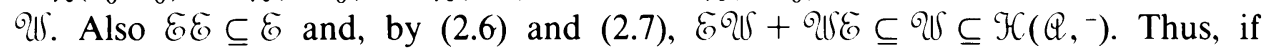
$w \in \mathcal{Q} S, s_{0} w=\overline{s_{0} w}=\bar{w} \bar{s}_{0}=-w s_{0}$. Hence if $w_{1}, w_{2} \in \mathcal{Q} \int, s_{0}\left(w_{1} w_{2}\right)=\left(s_{0} w_{1}\right) w_{2}-$ $\left[s_{0}, w_{1}, w_{2}\right]=-\left(w_{1} s_{0}\right) w_{2}+\left[w_{1}, s_{0}, w_{2}\right]=-w_{1}\left(s_{0} w_{2}\right)$. If we temporarily adopt the notation $\langle x, y\rangle=\chi(x, y)$, the argument on [1, pp. 153-154] can now be used word for word (omitting only the remarks establishing the nontriviality of the cubic forms) to obtain the required isomorphism.

Following [7], we call an element $e \in \mathbb{Q}$ strictly regular if $e \neq 0$ and $U_{e} x \in \mathfrak{f} e$ for $x \in \mathcal{Q}$. If $u \in Q$ is invertible, it follows from (1.13) that $e$ is strictly regular regarded as an element of $\left(Q,,^{-}\right)$if and only if $e$ is strictly regular regarded as an element of $\left(\mathbb{Q},{ }^{-}\right)^{\langle u\rangle}$. Also, if $\alpha$ is an isotopy of $\left(\mathbb{Q},,^{-}\right)$onto itself and $e \in \mathbb{Q}$ is strictly regular, it follows from (1.21) that $\alpha e$ is strictly regular. Finally, we observe that if $e \in Q$ is strictly regular, then $U_{e}$ is not invertible and hence $e$ is not invertible.

$\left(\mathbb{Q},{ }^{-}\right)$is said to be reduced if there is a strictly regular element in $\mathcal{G}$. The next proposition is an adaptation to our setting of results of Ferrar (see [7, Corollary 3.4 and Theorem 5.1]) on reduced simple Freudenthal triple systems.

THEOREM 4.6. The following statements are equivalent:

(i) $\left(Q,{ }^{-}\right)$is reduced.

(ii) There exists $u \in \mathbb{Q}$ so that $\mu \in \nu(u)^{\mathfrak{*} * 2}$.

(iii) $\left(\Theta,^{-}\right)$is isotopic to $\mathscr{T}\left(T, N, N^{\prime}\right)$ for some triple $\left(T, N, N^{\prime}\right)$ satisfying the adjoint identities.

Proof. Ferrar has shown [7, Corollary 3.4] that there exists $e \neq 0 \in \mathbb{Q}$ such that $(e, x, e)_{F} \in \mathcal{E} e$ for all $x \in \mathbb{Q}$ if and only if there exists $u \in \mathbb{Q}$ such that $\left\langle u,(u, u, u)_{F}\right\rangle_{F} \in 12 \mathfrak{f}^{* 2}$. In view of (2.16) and (2.17), this proves the equivalence of (i) and (ii).

Suppose (ii) holds. Then, $u$ is invertible and, in the notation of $\S 3, \mu^{\langle u\rangle}=\nu(u) \mu \in$ $\mathfrak{f}^{* 2}$. Thus, by Proposition 4.5, $\left(\mathbb{Q},{ }^{-}\right)^{\langle u\rangle} \cong \mathfrak{R}\left(T, N, N^{\prime}\right)$ for some triple $\left(T, N, N^{\prime}\right)$ satisfying the adjoint identities. Thus, we have (iii).

Conversely suppose (iii) holds. To prove (i), we may assume that $\left(\mathscr{Q},{ }^{-}\right)=$ T( $\left(T, N, N^{\prime}\right)$. But then it is easy to check that $\left[\begin{array}{ll}1 & 0 \\ 0 & 0\end{array}\right]$ is strictly regular. Thus, we have (i).

5. Extracting Jordan algebras of degree 3 from ones of degree 4. In this section, we are primarily interested in proving some facts about separable Jordan algebras of degree 4 . We can, however, consider a slightly more general situation.

If $Q$ is a form of degree 4 on a Jordan algebra $\mathscr{B}$ over we say that $Q$ is normalized if $Q(1)=1$, in which case we define the trace of $Q$ to be the symmetric bilinear form $\phi: \mathscr{B} \times \mathscr{B} \rightarrow k$ defined by

$$
\phi(b, c)=-\left.\partial_{b} \partial_{c} \log Q\right|_{1}=\left.\left.\partial_{b} Q\right|_{1} \partial_{c} Q\right|_{1}-\left.\partial_{b} \partial_{c} Q\right|_{1} .
$$


By a Jordan norm of degree 4 on a Jordan algebra $\mathscr{B}$ over $\mathfrak{f}$, we mean a normalized form $Q$ of degree 4 on $\mathscr{B}$ such that the trace form $\phi$ of $Q$ is nondegenerate and $Q\left(U_{a} b\right)=Q(a)^{2} Q(b)$ for all $a, b \in \mathscr{B}_{K}$ and all field extensions $K / \mathfrak{f}$. An example of a Jordan norm of degree 4 is the generic norm on a degree 4 separable Jordan algebra. Another example is the square of the generic norm on a separable degree 2 Jordan algebra. Using the results of [12], it is not difficult to list all possible Jordan norms of degree 4. However, we won't require such a list.

Suppose throughout the rest of the section that $Q$ is a Jordan norm of degree 4 on a Jordan algebra $\Re$ over $f$ and that $\phi$ is the trace form of $Q$. The product of $b_{1}$ and $b_{2}$ in $\Re$ will be denoted simply by $b_{1} b_{2}$. We write $\phi(b)=\phi(b, 1)$ for $b \in \Re$. Let $\lambda$ be transcendental over $\mathfrak{l}$. Put

$$
q_{b}(\lambda)=Q(\lambda 1-b)=\sum_{i=0}^{4} q_{i}(b) \lambda^{i}
$$

for $b \in \mathscr{B}$, where $q_{i}$ is a form of degree $4-i$ on $\mathscr{B}$ for $i=0, \ldots, 4, q_{4}=1$, and $q_{0}=Q$. The following properties are easily obtained using the results of [12 and 13]:

Proposition 5.1. (a) $\phi(1)=4$.

(b) $\phi$ is a nondegenerate symmetric associative bilinear form on $\Re$.

(c) $q_{b}(b)=0$ for $b \in \mathscr{B}$.

(d) $\Re$ is separable of degree $\leqslant 4$.

(e) If $b \in \Re$,

$$
\begin{aligned}
& q_{3}(b)=-\phi(b), \\
& q_{2}(b)=\frac{1}{2}\left(\phi(b)^{2}-\phi\left(b^{2}\right)\right), \\
& q_{1}(b)=\frac{1}{6}\left(3 \phi(b) \phi\left(b^{2}\right)-2 \phi\left(b^{3}\right)-\phi(b)^{3}\right), \\
& q_{0}(b)=\frac{1}{24}\left(3 \phi\left(b^{2}\right)^{2}+8 \phi(b) \phi\left(b^{3}\right)-6 \phi\left(b^{4}\right)-6 \phi(b)^{2} \phi\left(b^{2}\right)+\phi(b)^{4}\right) .
\end{aligned}
$$

(f) If $b \in \mathscr{B}$, then $b$ is invertible if and only if $Q(b) \neq 0$.

PROOF. Since a Jordan norm of degree 4 remains a Jordan norm of degree 4 under base field extension, we may assume that is infinite. Hence, $Q$ is a norm on $\mathscr{B}$ as defined on [12]. (a) follows from the Euler differential equation. In (b), only associativity needs to be proved and it follows from [12, Lemma 1.2]. (c) follows from [13, Lemma 2]. (d) follows from [12, Theorem 1.1] and (c). Next, to prove (e), we have by [13, Formula (6)], $\phi(d) q_{i}(b)=\left.\partial_{b d} q_{i}\right|_{b}-\left.\partial_{d} q_{i-1}\right|_{b}$ for $1 \leqslant i \leqslant 4$ and $b$, $d \in \Re$. If we set $d=b$ and use Euler's differential equation, we get

$$
(5-i) q_{i-1}(b)=\left.\partial_{b^{2}} q_{i}\right|_{b}-\phi(b) q_{i}(b) \text {. }
$$

(This recursion formula can be thought of as a version of Newton's identities.) Beginning with $q_{4}(b)=1$, we can solve successively for $q_{3}(b), q_{2}(b), q_{1}(b)$, and $q_{0}(b)$ and obtain (e). To prove (f), suppose $b \in \mathscr{B}$. If $b$ is invertible, $1=Q(1)=$ $Q\left(U_{b} b^{-2}\right)=Q(b)^{2} Q\left(b^{-2}\right)$ and so $Q(b) \neq 0$. Conversely, if $Q(b) \neq 0, b$ is invertible by (c). 
THEOREM 5.3. Suppose $\phi$ is the trace form of a Jordan norm $Q$ of degree 4 on a Jordan algebra $\Re_{\text {. Let }} \mathscr{G}_{0}=\left\{b \in \Re_{\mid} \mid \phi(b)=0\right\}$ and define $T: \mathscr{G}_{0} \times \mathscr{G}_{0} \rightarrow \mathfrak{f}$ and $N$ : $\mathscr{G}_{0} \rightarrow \mathfrak{f}$ by

$$
T(b, c)=\phi(b, c) \text { and } N(b)=\frac{1}{3} \phi\left(b^{3}\right)
$$

for $b, c \in 9_{0}$. Then, the triple $(T, N, N)$ defined on $\left(\mathscr{G}_{0}, \mathscr{T}_{0}\right)$ satisfies the adjoint identities. Moreover, $N=0$ if and only if either ${ }^{1} 1$ or $\mathscr{G}$ has degree 2 and $Q$ is the square of the generic norm on $\mathfrak{B}$.

Proof. Let $b, c \in \mathscr{G}_{0}$. Then, $b^{2}-\frac{1}{4} \phi\left(b^{2}\right) 1 \in \bigcap_{0}$ and $T\left(b^{2}-\frac{1}{4} \phi\left(b^{2}\right) 1, c\right)=$ $\phi\left(b^{2}, c\right)$. But since $N(b)=\frac{1}{3} \phi\left(b^{3}\right),\left.\partial_{c} N\right|_{b}=\frac{1}{3} \phi\left(b^{2} c+2 b(b c)\right)=\phi\left(b^{2}, c\right)$ (by the associativity of $\phi$ ). Hence, if \# : $\mathscr{G}_{0} \rightarrow \mathscr{G}_{0}$ is defined by (4.1), we have

$$
b^{\#}=b^{2}-\frac{1}{4} \phi\left(b^{2}\right) 1 .
$$

But then if we let $\equiv$ denote congruence modulo $\mathfrak{f}^{1}$, we have

$$
\begin{aligned}
\left(b^{\#}\right)^{\#} & \equiv\left(b^{2}-\frac{1}{4} \phi\left(b^{2}\right) 1\right)^{2} \equiv b^{4}-\frac{1}{2} \phi\left(b^{2}\right) b^{2}=b^{4}+q_{3}(b) b^{3}+q_{2}(b) b^{2} \\
& \equiv-q_{1}(b) b \quad(\text { by }(5) \\
& =\frac{1}{3} \phi\left(b^{3}\right) b \quad(\text { by }(5.2)) \\
& =N(b) b
\end{aligned}
$$

for $b \in \mathscr{G}_{0}$. Thus, $\left(b^{\#}\right)^{\#}=N(b) b$ and we have proved the first statement.

For the second statement we may assume that $\mathscr{h} \neq \mathfrak{f} 1$. Let $n$ and $t$ denote the generic norm and trace on $\mathscr{B}$, respectively. If $\mathscr{B}$ has degree 2 and $Q=n^{2}$, then $\phi=2 t$ and so if $b \in \mathfrak{H}_{0}, N(b)=\frac{2}{3} t\left(b, b^{2}\right)=\frac{2}{3} t(b, t(b) b-n(b) 1)=0$. Conversely, suppose that $N=0$. Then $\phi\left(b^{3}\right)=0$ for $b \in \mathscr{B}_{0}$. Polarizing gives $\phi\left(b^{2}, c\right)=0$ for $b, c \in \mathscr{H}_{0}$. Hence, $b^{2} \in \mathfrak{l} 1$ for $b \in \mathscr{B}_{0}$. But if $b \in \mathscr{B}, b-\frac{1}{4} \phi(b) \in \mathscr{G}_{0}$, and so $\left(b-\frac{1}{4} \phi(b) 1\right)^{2} \in \mathfrak{f} 1$. Thus, $b^{2}-\frac{1}{2} \phi(b) b \in \mathfrak{t} 1$ for $b \in \mathscr{B}$. Hence, $\Re$ has degree 2 . Thus, $b^{2}-t(b) b \in \mathfrak{f} 1$ and hence $\left(t(b)-\frac{1}{2} \phi(b)\right) b \in \mathfrak{l} 1$ for $b \in \Re$. Therefore, $\phi=2 t$. But by (5.2) a Jordan norm of degree 4 on $\mathscr{B}$ is completely determined by its trace form. Therefore, $Q=n^{2}$.

THEOREM 5.4. Suppose $\mathfrak{B}$ is a degree 4 separable Jordan algebra with generic trace $t$. Let $\mathscr{B}_{0}=\{b \in \Re \mid t(b)=0\}$ and choose $e \in \mathscr{B}_{0}$ such that $t\left(e^{3}\right) \neq 0$. Then, $\mathscr{\Re}_{0}$ has the unique structure of a separable degree 3 Jordan algebra with identity $e$ and generic norm $N_{\mathscr{B}_{0}}$ given by

$$
N_{G_{B_{0}}}(b)=\frac{t\left(b^{3}\right)}{t\left(e^{3}\right)} .
$$

Moreover, if $\mathscr{G}_{3}$ is central simple, then $\mathscr{B}_{0}$ is central simple.

Proof. Let $Q=n$, where $n$ is the generic norm on $\mathscr{B}$. Then, $Q$ is a Jordan norm of degree 4 on $\mathscr{B}$ with trace form $\phi=t$. Define $T(b, c)=t(b, c)$ and $N(b)=\frac{1}{3} t\left(b^{3}\right)$ for $b \in \mathscr{G}_{0}$. Then, the triple $(T, N, N)$ satisfies the adjoint identities and is nontrival. 
Moreover, $\operatorname{dim} \Re_{0} \geqslant 3$, and so by the results of [17 and 18], $\mathscr{G}_{0}$ has the structure of a separable degree 3 Jordan algebra with identity $e$ and generic norm $N_{\mathscr{C}_{0}}$ given by $N_{\mathscr{G}_{0}}(b)=N(b) / N(e)$. The uniqueness of this structure is well known and follows from [10, Theorem 6.7]. To prove the last statement, we may assume that $\mathfrak{f}$ is algebraically closed. Thus, we may identify $\mathscr{B}$ with the Jordan algebra of all $4 \times 4$ hermitian matrices over an associative composition algebra. In order to prove that $\mathscr{B}_{0}$ is central simple, it is sufficient to prove that $N$ is irreducible. Suppose the contrary. Then, the restriction of $N$ to any subspace of $\mathscr{G}_{0}$ is either zero or reducible. Denote the matrix units by $e_{i j}, 1 \leqslant i, j \leqslant 4$. If

$$
b=\xi_{11}\left(e_{11}-e_{33}\right)+\xi_{22}\left(e_{22}-e_{33}\right)+\sum_{1 \leqslant i \leqslant j \leqslant 3} \xi_{i j}\left(e_{i j}+e_{j i}\right),
$$

where $\xi_{i j} \in \mathfrak{f}$ for all $i, j$, then

$$
N(b)=-\xi_{11}^{2} \xi_{22}-\xi_{11} \xi_{22}^{2}-\xi_{13}^{2} \xi_{22}-\xi_{11} \xi_{23}^{2}+\xi_{11} \xi_{12}^{2}+\xi_{22} \xi_{12}^{2}+2 \xi_{12} \xi_{23} \xi_{13} .
$$

This polynomial is easily seen to be irreducible yielding a contradiction.

COROLlary 5.5. Suppose $\mathscr{B}$ is a 28-dimensional central simple Jordan algebra of degree 4 with generic trace $t$. Let $\mathscr{B}_{0}=\left\{b \in \Re_{\mid c} t(b)=0\right\}$ and choose $e \in \mathscr{B}_{0}$ such that $t\left(e^{3}\right) \neq 0$. Then, $\mathscr{B}_{0}$ has the unique structure of a 27-dimensional exceptional central simple Jordan algebra with identity $e$ and generic norm given by $N_{\mathscr{B}_{0}}(b)=$ $t\left(b^{3}\right) / t\left(e^{3}\right)$.

Proof. Any 27-dimensional central simple Jordan algebra of degree 3 is exceptional [10, Corollary 5.2].

The following proposition shows that every degree 3 separable Jordan algebra $q$ can be obtained using the process described in Theorem 5.4 beginning with the (nonsimple) algebra $\mathscr{B}=\mathfrak{i} \oplus \mathscr{g}$.

Proposition 5.6. Suppose $\mathcal{G}$ is a degree 3 separable Jordan algebra with generic norm $N_{g}$, generic trace $T_{g}$, and identity $1 \mathcal{g}$. Let $\mathfrak{B}=\mathfrak{l} \oplus \mathcal{G}($ as algebras $), e=\left(\frac{3}{2},-\frac{1}{2} 1_{g}\right)$, and let $t$ be the generic trace on 9 . Then, $t\left(e^{3}\right)=3$ and (using the notation of Theorem 5.4) the map $j \rightarrow \tilde{j}=\left(\frac{1}{2} T_{g}(j), j-\frac{1}{2} T_{g}(j) 1_{g}\right)$ is a linear bijection on $g$ onto $\Re_{0}$ such that $N_{\mathfrak{B}_{0}}(\tilde{j})=N_{\mathfrak{g}}(j)$ for $j \in \mathcal{g}$.

Proof. Now $t(\alpha, j)=\alpha+T_{g}(j)$ for $\alpha \in \mathfrak{f}, j \in \mathcal{g}$. Hence, $t\left(e^{3}\right)=t\left(\frac{27}{8},-\frac{1}{8} 1_{g}\right)$ $=\frac{27}{8}-\frac{3}{8}=3$. Also $\mathscr{B}_{0}=\left\{\left(-T_{\mathfrak{g}}(j), j\right) \mid j \in \mathcal{g}\right\}$. If $j \in \mathcal{g}$, we have

$N_{\mathscr{B}_{0}}\left(\left(-T_{g}(j), j\right)\right)=\frac{1}{3} t\left(\left(-T_{g}(j)^{3}, j^{3}\right)\right)=\frac{1}{3}\left(T_{g}\left(j^{3}\right)-T_{g}(j)^{3}\right)=N_{g}\left(j-T_{g}(1) 1_{g}\right)$.

(The last equality is easily checked using $6 N_{g}(j)=-3 T_{g}(j) T_{g}\left(j^{2}\right)+2 T_{g}\left(j^{3}\right)+$ $T_{g}(j)^{3}$ which is proved in the same way as (5.2).) But the map $\left(-T_{g}(j), j\right) \rightarrow j-$ $T_{g}(j) l_{g}$ of $\Re_{0}$ into $g$ has inverse $j \rightarrow \tilde{j}$ and so we have proved the proposition.

An alternate description of the algebra $\mathscr{B}$ in Proposition 5.6 can be given. Suppose we have the hypotheses and notation of Proposition 5.6. Let $\chi: \mathcal{g} \times \mathcal{g} \rightarrow \mathcal{g}$ be defined as usual by

$$
j \times k=2 j \cdot k-T_{g}(j) k-T_{g}(k) j+\left(T_{g}(j) T_{\mathfrak{g}}(k)-T_{\mathfrak{g}}(j, k)\right) \mathrm{l}_{\mathfrak{g}},
$$


where $\cdot$ is the product on $q$. Then, one easily checks that

$$
\tilde{j k}=\frac{1}{4} T_{\mathfrak{q}}(j, k) 1_{\mathfrak{G}}+\frac{1}{2}(j \times k)^{\sim}
$$

for $j, k \in \mathcal{G}$, where $1_{\mathfrak{C B}}=\left(1,1_{\mathcal{G}}\right)$. Then, $\mathscr{B}=\mathfrak{k} 1_{\mathfrak{C B}} \oplus \mathscr{B}_{0}$ (as vector spaces), $1_{\mathfrak{G}}$ is the identity of $\mathscr{B}$, and the multiplication on $\mathfrak{B}_{0}=\{\tilde{j} \mid j \in \mathcal{G}\}$ is given by (5.7). Finally, $e=\tilde{1}_{g}$.

6. The Cayley-Dickson process. Suppose $\left(\Re,{ }^{-}\right)$is an algebra with involution over $\mathfrak{f}$ and suppose $\phi: \mathbb{Q} \times \mathbb{Q} \rightarrow \mathfrak{H}$ is a symmetric $\mathfrak{f}$-bilinear form on $\mathbb{Q}$ such that $\phi(1,1) \neq 0$ and $\phi(b, 1)=\phi(\bar{b}, 1)$ for all $b \in \mathscr{B}$. We write $\phi(b)=\phi(b, 1)$ for $b \in \mathscr{B}$. Define $\theta$ : $\mathscr{B} \rightarrow \mathscr{B}$ by

$$
b^{\theta}=-b+\frac{2 \phi(b)}{\phi(1)} 1 .
$$

Then, $\theta$ is a ${ }^{-}$-linear bijection of order 2 such that $\bar{b}^{\theta}=\overline{b^{\theta}}$ and $\phi\left(b_{1}^{\theta}, b_{2}^{\theta}\right)=\phi\left(b_{1}, b_{2}\right)$ for $b, b_{1}, b_{2} \in \mathscr{B}$. Let $\mu \neq 0 \in \mathfrak{f}$. We construct a new algebra with involution ( $Q$,,$\left.^{-}\right)$ over $\mathfrak{A}$ as follows: Let $\mathscr{Q}=\mathscr{B} \oplus \mathscr{B}$ (as vector spaces). Define a multiplication on $\mathscr{Q}$ by

$$
\left(b_{1}, b_{2}\right)\left(b_{3}, b_{4}\right)=\left(b_{1} b_{3}+\mu\left(b_{2} b_{4}^{\theta}\right)^{\theta}, b_{1}^{\theta} b_{4}+\left(b_{2}^{\theta} b_{3}^{\theta}\right)^{\theta}\right) .
$$

Define $\left(\overline{b_{1}, b_{2}}\right)=\left(\bar{b}_{1},-\bar{b}_{2}^{\theta}\right)$. Using the fact that $\theta$ commutes with ${ }^{-}$, it is straightforward to check that $\left(Q,,^{-}\right)$is an algebra with involution. We identify $b \in \mathscr{B}$ with $(b, 0) \in \mathbb{Q}$. Then, $\left(\mathscr{G},{ }^{-}\right)$is a subalgebra of $\left(\mathbb{Q},{ }^{-}\right)$. Let $s_{0}=(0,1)$. Then, $(0, b)=s_{0} b$ for $b \in \mathscr{B}$. Hence, if $b_{1}, b_{2} \in \mathfrak{B},\left(b_{1}, b_{2}\right)=b_{1}+s_{0} b_{2}$ and so $\mathcal{Q}=\mathscr{B} \oplus s_{0} \mathscr{B}$. We can extend $\phi$ to a symmetric $\mathfrak{f}^{-}$-bilinear form (also denoted by $\phi$ ) on $\mathfrak{Q}$ by defining

$$
\phi\left(b_{1}+s_{0} b_{2}, b_{3}+s_{0} b_{4}\right)=\phi\left(b_{1}, b_{3}\right)-\mu \phi\left(b_{2}, b_{4}\right) .
$$

In that case the extended form also satisfies $\phi(1,1) \neq 0$ and $\phi(\bar{a}, 1)=\phi(a, 1)$ for $a \in \mathcal{Q}$. We call the process described in this paragraph the (generalized) CayleyDickson process. Although we will not need this fact, it is easily checked that if the form $\phi$ on $\Re$ satisfies the equations

$$
\phi(\bar{x}, \bar{y})=\phi(x, y) \text { and } \phi(x y, z)=\phi(y, \bar{x} z)
$$

for $x, y, z \in \mathscr{B}$, then the extended form $\phi$ on $\mathcal{Q}$ satisfies the same equations for $x, y$, $z \in \mathbb{Q}$.

If $(\mathscr{B},-)$ is an algebra with involution over $\mathfrak{t}$ satisfying $b+\bar{b} \in \mathfrak{t} 1$ and $b \bar{b} \in \mathfrak{t} 1$ for all $b \in \mathscr{B}$, then we can define $\phi: \mathcal{B} \times \mathscr{B} \rightarrow \mathfrak{f}$ by $b_{1} \bar{b}_{2}+b_{2} \bar{b}_{1}=\phi\left(b_{1}, b_{2}\right) 1$. In that case $b^{\theta}=\bar{b}$ for $b \in \mathscr{B}$ and the above process is the classical Cayley-Dickson process $[15$, p. 45$]$. We are interested here in a different special case.

Suppose for the rest of the section that $\mu \neq 0 \in \mathfrak{f}$ and that $Q$ is a Jordan norm of degree 4 on a Jordan algebra $\mathscr{B}$ over $\mathfrak{k}$ and that $\phi$ is the trace form of $Q$. We regard $B$ as an algebra with involution - by taking - to be the identity map on $\mathfrak{B}$. Since $\phi(1)=4$, the map $\theta$ is given by

$$
b^{\theta}=-b+\frac{1}{2} \phi(b) 1
$$

for $b \in \mathfrak{B}$. We have

$$
C D(\mathscr{B}, Q, \mu)=\mathscr{B} \oplus s_{0} \mathscr{B}
$$


In fact every element of $C D(\Re, Q, \mu)$ is uniquely expressible in the form $b_{1}+s_{0} b_{2}$, where $b_{1}, b_{2} \in \mathscr{B}$. The multiplication and involution on $C D(\Re, Q, \mu)$ are given by

$$
\left(b_{1}+s_{0} b_{2}\right)\left(b_{3}+s_{0} b_{4}\right)=b_{1} b_{3}+\mu\left(b_{2} b_{4}^{\theta}\right)^{\theta}+s_{0}\left(b_{1}^{\theta} b_{4}+\left(b_{2}^{\theta} b_{3}^{\theta}\right)^{\theta}\right)
$$

and

$$
\overline{b_{1}+s_{0} b_{2}}=b_{1}-s_{0} b_{2}^{\theta} \text {. }
$$

Put $\mathscr{\Re}_{0}=\{b \in \mathscr{B} \mid \phi(b)=0\}$. Then, by (6.4), $\delta=\delta(C D(\mathscr{B}, Q, \mu))=\mathfrak{f} s_{0}$ and $\mathcal{H}=\mathcal{H}(C D(\mathscr{B}, Q, \mu))=\mathscr{B} \oplus s_{0} \Re_{0}$.

Proposition 6.5. Suppose $\mu \in \mathfrak{f}^{* 2}$. Then, $C D(\mathscr{B}, Q, \mu) \cong \mathfrak{R}(T, N, N)$, where $(T, N, N)$ is the triple defined on $\left(\Re_{0}, \Re_{0}\right)$ by $T(b, c)=\phi(b, c)$ and $N(b)=\frac{1}{3} \phi\left(b^{3}\right)$ for $b, c \in \Re_{0}$.

Proof. Define $\eta: C D(\Re, Q, \mu) \rightarrow \mathfrak{T}(T, N, N)$ by

$$
\eta\left(\alpha 1+b+s_{0}(\beta 1+c)\right)=\left[\begin{array}{ll}
\alpha+\sqrt{\mu} \beta & \frac{1}{2}(b+\sqrt{\mu} c) \\
\frac{1}{2}(b-\sqrt{\mu} c) & \alpha-\sqrt{\mu} \beta
\end{array}\right]
$$

for $\alpha, \beta \in \mathfrak{f}, b, c \in \Re_{0}$. A straightforward calculation shows that $\eta$ is an isomorphism.

THEOREM 6.6. Suppose $\mu \in \mathfrak{l}^{*}$ and $Q$ is a Jordan norm of degree 4 on a Jordan algebra $\Re$ over $\mathfrak{1}$. Then, $C D(\mathscr{B}, Q, \mu)$ is a simple structurable algebra with $\operatorname{dim}_{\mathrm{f}} \delta(C D(\Re, Q, \mu))=1$.

Proof. By extending the base field, we may assume that $\mu \in \mathfrak{f}^{* 2}$. But then the conclusion follows from the corresponding fact for the matrix algebra $\Re(T, N, N)$ described in the statement of Proposition 6.5.

Thus, $C D(\Re, Q, \mu)$ is an algebra with involution satisfying the hypotheses of $\S 2$. Let $\chi=\chi_{C D(\mathscr{B}, Q, \mu)}$ and $\nu=\nu_{C D(\mathscr{B}, Q, \mu)}$.

Proposition 6.7. If $b_{1}, b_{2}, b_{3}, b_{4} \in \mathscr{B}$, then

$$
\chi\left(b_{1}+s_{0} b_{2}, b_{3}+s_{0} b_{4}\right)=\phi\left(b_{1}, b_{3}\right)-\mu \phi\left(b_{2}, b_{4}\right)
$$

and

$$
\nu\left(b_{1}+s_{0} b_{2}\right)=Q\left(b_{1}\right)+\mu^{2} Q\left(b_{2}\right)+\frac{\mu}{2} \phi\left(U_{b_{1}} b_{2}, b_{2}\right)-\frac{\mu}{4} \phi\left(b_{1}, b_{2}\right)^{2}
$$

Proof. If $b \in \Re$,

$$
\begin{aligned}
\mu \chi(b, 1) & =2 \psi\left(s_{0} b, 1\right) s_{0} \quad(\text { by }(2.3)) \\
& =2\left(s_{0} b-\overline{s_{0} b}\right) s_{0} \\
& =2\left(s_{0} b+s_{0} b^{\theta}\right) s_{0}=\phi(b) s_{0}^{2}=\mu \phi(b)
\end{aligned}
$$

and so

$$
\chi\left(b_{1}, b_{3}\right)=\chi\left(b_{1} b_{3}, 1\right)=\phi\left(b_{1} b_{3}\right)=\phi\left(b_{1}, b_{3}\right) \quad(\text { by }(2.7)) .
$$


Also

$$
\chi\left(b_{1}, s_{0} b_{4}\right)=\chi\left(b_{1} b_{4}, s_{0}\right)=0 \quad(\text { by }(2.6) \text { and }(2.7))
$$

and

$$
\chi\left(s_{0} b_{2}, s_{0} b_{4}\right)=-\chi\left(b_{2}, s_{0}\left(s_{0} b_{4}\right)\right)=-\mu \chi\left(b_{2}, b_{4}\right)=-\mu \phi\left(b_{2}, b_{4}\right) \quad(\text { by }(2.7))
$$

and we have proved (6.8).

Next if $b \in \Re$, we have $12 \mu \nu(b)=\chi\left(s_{0} b,\left\{b, s_{0} b, b\right\}\right)$ (by (2.10)) and

$$
\begin{aligned}
\left\{b, s_{0} b, b\right\} & =2\left(b\left(\overline{s_{0} b}\right)\right) b-(b \bar{b})\left(s_{0} b\right)=-2\left(b\left(s_{0} b^{\theta}\right)\right) b-b^{2}\left(s_{0} b\right) \\
& =-s_{0}\left(2\left(b^{\theta 2 \theta} b^{\theta}\right)^{\theta}+b^{2 \theta} b\right) .
\end{aligned}
$$

Hence, by (6.8),

$$
12 \nu(b)=\phi\left(b, 2\left(b^{\theta 2 \theta} b^{\theta}\right)^{\theta}+b^{2 \theta} b\right) .
$$

But using (6.1), it is easy to check that $\phi\left(b_{1}^{\theta}, b_{2}^{\theta}\right)=\phi\left(b_{1}, b_{2}\right)$. Hence $12 \nu(b)=$ $2 \omega\left(b^{\theta}\right)+\omega(b)$, where $\omega(b)=\phi\left(b^{2}, b^{2 \theta}\right)$. But

$$
\omega(b)=\phi\left(b^{2},-b^{2}+\frac{1}{2} \phi\left(b^{2}\right) 1\right)=-\phi\left(b^{4}\right)+\frac{1}{2} \phi\left(b^{2}\right)^{2}
$$

and so $2 \omega\left(b^{\theta}\right)=-2 \phi\left(b^{\theta 4}\right)+\phi\left(b^{\theta 2}\right)^{2}$. If we use (6.1) and simplify using $\phi(1)=4$, we get

$$
2 \omega\left(b^{\theta}\right)=-2 \phi\left(b^{4}\right)+4 \phi(b) \phi\left(b^{3}\right)-3 \phi(b)^{2} \phi\left(b^{2}\right)+\frac{1}{2} \phi(b)^{4}+\phi\left(b^{2}\right)^{2}
$$

and so

$12 \nu(b)=-3 \phi\left(b^{4}\right)+4 \phi(b) \phi\left(b^{3}\right)-3 \phi(b)^{2} \phi\left(b^{2}\right)+\frac{1}{2} \phi(b)^{4}+\frac{3}{2} \phi\left(b^{2}\right)^{2}=12 q_{0}(b)$ (by (5.2)). Therefore, $\nu(b)=q_{0}(b)=Q(b)$ for $b \in \mathscr{B}$.

Next by $(6.8), \chi\left(\mathscr{B}, s_{0} \mathscr{B}\right)=\{0\}$. Also, by (6.3), $\left\{s_{0} \mathscr{\Re}, \mathscr{G}, s_{0} \mathscr{G}\right\} \subseteq \mathscr{G}$ and $\left\{\mathfrak{B}, s_{0} \mathscr{\Re}, \mathscr{B}\right\} \subseteq s_{0} \mathscr{B}$. Hence it follows from (2.15) that $\nu\left(b_{1}, s_{0} b_{2}, s_{0} b_{2}, s_{0} b_{2}\right)=0$ and $\nu\left(s_{0} b_{2}, b_{1}, b_{1}, b_{1}\right)=0$. Thus

$$
\begin{aligned}
\nu\left(b_{1}+s_{0} b_{2}\right) & =\nu\left(b_{1}\right)+\frac{1}{4} \nu\left(s_{0} b_{2}, b_{1}, s_{0} b_{2}, b_{1}\right)+\nu\left(s_{0} b_{2}\right) \\
& =Q\left(b_{1}\right)+\frac{1}{4} \nu\left(s_{0} b_{2}, b_{1}, s_{0} b_{2}, b_{1}\right)+\mu^{2} Q\left(b_{2}\right)
\end{aligned}
$$

(by (3.10)). Finally,

$$
\begin{aligned}
\nu\left(s_{0} b_{2}, b_{1}, s_{0} b_{2}, b_{1}\right) & =2 \mu \chi\left(b_{2},\left\{b_{1}, b_{2}, b_{1}\right\}\right)-\mu \chi\left(b_{2}, b_{1}\right)^{2} \\
& =2 \mu \phi\left(b_{2}, U_{b_{1}} b_{2}\right)-\mu \phi\left(b_{1}, b_{2}\right)^{2}
\end{aligned}
$$

and we have proved (6.9).

According to (6.8), $\chi$ is the extension discussed at the beginning of this section of $\phi$ to $C D(\Re, Q, u)$.

Corollary 6.10. Let $c \in \mathscr{B}$. Then, $c$ is invertible as an element of $\left(\mathbb{Q},,^{-}\right)$if and only if $c$ is invertible as an element of $\Re$. Moreover if $c$ is invertible, then $\hat{c}=c^{-1}$ and

$$
P_{c}\left(b_{1}+s_{0} b_{2}\right)=U_{c} b_{1}+Q(c) s_{0} U_{c}^{-1} b_{2}
$$

for $b_{1}, b_{2} \in \mathcal{\delta}$. 
Proof. The first statement follows from (6.9), Corollary 2.11, and Proposition 5.1(f). Suppose $c$ is invertible. Then, $U_{c} c^{-1}=c$ and hence $c^{-1}=\hat{c}$. Next if $b \in \Re$, $\tau^{\langle c\rangle} b=2 b-\left\{b, c, c^{-1}\right\}=2 b-\left\{c^{-1}, c, b\right\}=b$ and so $P_{c} b=U_{c} b$. Finally, $P_{c}$ is an isotopy of $\left(Q,^{-}\right)$and so $\left(P_{c}\right)_{\S}\left(s_{0}\right)=\gamma s_{0}$ for some $\gamma \neq 0 \in \mathfrak{f}$. Thus, for $b \in \mathscr{B}$, $P_{c}\left(s_{0} b\right)=\gamma s_{0} \hat{P}_{c} b$ (by $\left.(1.22)\right)=\gamma s_{0} P_{c}^{-1} b=\gamma s_{0} U_{c}^{-1} b$. It remains to show that $\gamma=$ $Q(c)$. But $P_{c}\left(s_{0} c^{2}\right)=\gamma s_{0} U_{c}^{-1} c^{2}=\gamma s_{0}$. Multiplying both sides on the left by $s_{0}$ gives $\gamma \mu 1=s_{0} P_{c}\left(s_{0} c^{2}\right)=\mu \nu(c) P_{c}^{-1} c^{2}$ (by (3.4)) $=\mu Q(c) 1$ (by (6.9)).

EXAMPLE 6.11. Let

$$
S=\operatorname{diag}\left\{\left[\begin{array}{rr}
0 & 1 \\
-1 & 0
\end{array}\right],\left[\begin{array}{rr}
0 & 1 \\
-1 & 0
\end{array}\right],\left[\begin{array}{rr}
0 & 1 \\
-1 & 0
\end{array}\right],\left[\begin{array}{rr}
0 & 1 \\
-1 & 0
\end{array}\right]\right\}
$$

and let $\mathscr{B}$ be the Jordan algebra of all $8 \times 8$ matrices $A$ over ${ }^{f}$ such that $S^{-1} \circ A^{t} \circ S$ $=A$, where $\circ$ denotes the usual matrix product. Then, $\mathscr{B}$ is a central simple Jordan algebra of degree 4. The maps $A \rightarrow S \circ A$ and $A \rightarrow A \circ S$ are linear bijections of $\mathscr{B}$ onto the vector space of all skew-symmetric $8 \times 8$-matrices, and the generic norm $n$ and generic trace $t$ on $\mathscr{B}$ are given by

$$
n(A)=\operatorname{Pf}(S \circ A)=\operatorname{Pf}(A \circ S) \text { and } t(A)=\frac{1}{2} \operatorname{tr}(A)
$$

for $A \in \mathscr{B}$, where $\operatorname{Pf}(C)$ denotes the Pfaffian of the skew-symmetric matrix $C$ (see $[10, \S 6.4])$. Let $(\mathscr{Q},-)=C D(\Re, n, \mu)$, where $\mu \in \mathfrak{L}^{*}$, and put $\nu=\nu_{(\mathscr{Q},-)}$. Then, if $x=b_{1}+s_{0} b_{2} \in \mathbb{Q}$,

$$
\begin{aligned}
\nu(x) & =n\left(b_{1}\right)+\mu^{2} n\left(b_{2}\right)+\frac{\mu}{2} t\left(b_{1} \circ b_{2} \circ b_{1}, b_{2}\right)-\frac{\mu}{4} t\left(b_{1}, b_{2}\right)^{2} \quad(\text { by }(6.9)) \\
& =\operatorname{Pf}\left(b_{1} \circ S\right)+\mu^{2} \operatorname{Pf}\left(S \circ b_{2}\right)+\frac{\mu}{4} \operatorname{tr}\left(b_{1} \circ b_{2} \circ b_{1} \circ b_{2}\right)-\frac{\mu}{16}\left(\operatorname{tr}\left(b_{1} \circ b_{2}\right)\right)^{2} .
\end{aligned}
$$

Since $S^{-1}=-S$, each element $x \in \mathbb{Q}$ can be written in the form

$$
x=X \circ S+s_{0} S \circ Y,
$$

where $X, Y$ are skew-symmetric $8 \times 8$-matrices. In that case,

$$
\nu(x)=\operatorname{Pf}(X)+\mu^{2} \operatorname{Pf}(Y)+\frac{\mu}{4} \operatorname{tr}(X \circ Y \circ X \circ Y)-\frac{\mu}{16}(\operatorname{tr}(X \circ Y))^{2} .
$$

If $\mu=-1$, this is the quartic form used by Freudenthal in [8] to construct the complex simple Lie algebra of type $E_{7}$.

EXAMPLE 6.12. Suppose more generally that $\mathscr{B}$ is a central simple Jordan algebra of dimension $d$ and degree 4 over $¥$. Then, $d=10,16$, or 28 . Let $n$ and $t$, respectively, be the generic norm and trace on $\mathscr{B}$. Suppose $\mu \in \mathfrak{f}^{*}$. Let $\overline{\mathfrak{f}}$ be the

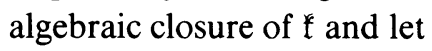

$$
g=\left(\Re_{\overline{\mathrm{f}}}\right)_{0}=\left\{b \in \mathscr{B}_{\overline{\mathrm{f}}} \mid t(b)=0\right\} .
$$

Then,

$$
C D(\mathscr{B}, n, \mu)_{\overline{\mathrm{f}}} \cong C D\left(\mathscr{B}_{\mathfrak{f}}, n, \mu\right) \cong \Re(T, N, N)
$$

(by Proposition 6.5), where $T: \mathcal{g} \times \mathcal{F} \rightarrow \overline{\mathfrak{f}}$ and $N: \mathcal{G} \rightarrow \mathfrak{f}$ are defined by $T(b, c)=$ $t(b, c)$ and $N(b)=\frac{1}{3} t\left(b^{3}\right)$. Choose $e \in \mathcal{G}$, so that $t\left(e^{3}\right) \neq 0$. Then, it follows from the results of [17 and 18] that $q$ has the structure of a separable Jordan algebra of degree 3 over $\bar{f}$ with identity $e$ and generic norm $N_{q}$ given by $N_{g}(b)=\zeta^{-1} N(b)$, where 
$\zeta=N(e)$. Moreover, the triple $(T, N, N)$ on $(q, g)$ is isomorphic to $\left(\zeta T_{q}, \zeta N_{q}, \zeta^{2} N_{q}\right)$ on $(\mathcal{g}, \mathcal{g})$, where $T_{g}$ is the generic trace on $\mathcal{g}$. Thus,

$$
C D(\mathscr{B}, n, \mu)_{\overline{\mathrm{f}}} \cong \Re\left(\zeta T_{\mathrm{g}}, \zeta N_{g}, \zeta^{2} N_{g}\right) .
$$

But if we choose a cube root $\gamma$ of $\zeta$ in $\bar{f}$, then the map

$$
\left[\begin{array}{cc}
\alpha & j \\
k & \beta
\end{array}\right] \rightarrow\left[\begin{array}{cc}
\alpha & \gamma j \\
\gamma^{2} k & \beta
\end{array}\right]
$$

is easily checked to be an isomorphism of $\Re\left(\zeta T_{\mathcal{g}}, \zeta \dot{N}_{\mathfrak{g}}, \zeta^{2} N_{\mathfrak{g}}\right)$ onto $\Re\left(T_{\mathcal{g}}, N_{\mathfrak{g}}, N_{\mathcal{g}}\right)$. Hence, $C D(\mathscr{B}, n, \mu)_{\bar{f}} \cong \mathscr{R}\left(T_{\mathfrak{g}}, N_{\mathfrak{g}}, N_{\mathfrak{g}}\right)$. Finally, by Corollary $5.4, g$ is simple. Thus, $C D(\mathscr{B}, n, \mu)_{\mathfrak{q}} \cong \Re\left(T_{g}, N_{g}, N_{g}\right)$, where $T_{g}$ and $N_{g}$ are, respectively, the generic trace and norm on the simple Jordan algebra of dimension $d-1$ and degree 3 over $\bar{f}$.

7. Division algebras. If $\left(Q,^{-}\right)$is a central simple structurable algebra over $\mathfrak{f}$, a central simple Z-graded Lie algebra $K\left(\mathbb{Q},,^{-}\right)$can be constructed from $\left(\mathbb{Q},{ }^{-}\right)$using a construction of Kantor (see [2]). If char $\mathfrak{t}=0$, it is not difficult to show that the Lie algebra $\mathcal{K}(\mathcal{Q},-)$ has relative rank 1 if and only if $(\mathscr{Q},-)$ is a division algebra. (The relative rank of a central simple Lie algebra is the dimension of a maximal split toral subalgebra [16]). Thus, from the point of view of Lie algebras it is of interest to construct central simple structurable division algebras. The following result shows that such algebras arise from the Cayley-Dickson process for certain choices of $\mathfrak{B}, Q$ and $\mu$.

THEOREM 7.1. Suppose $\mathfrak{k}=F(\xi)$ where $\xi$ is transcendental over a field $F$, and suppose $\mathscr{B}=\mathcal{C} \otimes_{F} \mathfrak{k}$, where $\mathcal{C}$ is a Jordan division algebra over $F$. Suppose $Q$ is the extension to $\mathscr{B}$ of a Jordan norm of degree 4 on $\mathcal{C}$. Then, $C D(\mathscr{B}, Q, \xi)$ is a central simple structurable division algebra over $\mathrm{f}$.

Proof. Let $\left(\mathscr{Q},,^{-}\right)=C D(\mathscr{B}, Q, \xi)$ and $\nu=\nu_{(\mathscr{Q},-)} .\left(\mathscr{Q},,^{-}\right)$is a central simple structurable algebra by Theorem 6.6 and Lemma 2.1(c). Suppose for contradiction that $(\mathbb{Q},-)$ is not a division algebra. Thus, by Proposition 2.11, there exists $x \neq 0 \in \mathbb{Q}$ so that $\nu(x)=0$. Then, $x=b_{1}+s_{0} b_{2}$, where $b_{1}, b_{2} \in \mathfrak{B}$. Let $\left(\nu_{1}, \ldots, v_{n}\right)$ be a basis for $C$ over $F$. Then, $b_{1}=\Sigma f_{i}(\xi) v_{i}$ and $b_{2}=\Sigma g_{i}(\xi) v_{i}$, where $f_{i}(\xi), g_{i}(\xi) \in F(\xi)$. Replacing $x$ by a nonzero $F(\xi)$ multiple of $x$, we may assume that $f_{i}(\xi), g_{i}(\xi) \in F[\xi]$ for all $i$ and that either $\xi \nmid f_{j}(\xi)$ for some $j$ or $\xi \nmid g_{j}(\xi)$ for some $j$. Next

$$
0=\nu(x)=Q\left(b_{1}\right)+\xi^{2} Q\left(b_{2}\right)+\frac{\xi}{2} \phi\left(U_{b_{1}} b_{2}, b_{2}\right)-\frac{\xi}{4} \phi\left(b_{1}, b_{2}\right)^{2} .
$$

Setting $\xi=0$, gives $0=Q\left(b_{1}(0)\right)$, where $b_{1}(0)=\Sigma f_{i}(0) v_{i}$. Thus, by Proposition 5.1(f), $b_{1}(0)=0$ and so $\xi \mid f_{i}(\xi)$ for all $i$. Therefore, $\xi \nmid g_{j}(\xi)$ for some $j$. Write $f_{i}(\xi)=\xi h_{i}(\xi)$, where $h_{i}(\xi) \in F[\xi]$ for $i=1, \ldots, n$. Put $b_{3}=\Sigma h_{i}(\xi) v_{i}$. Then, $x=$ $\xi b_{3}+s_{0} b_{2}$. Hence, $s_{0} x=\xi b_{2}+s_{0} \xi b_{3}=\xi y$, where $y=b_{2}+s_{0} b_{3}$. But $\nu(y)=$ $\nu\left(\xi^{-1} s_{0} x\right)=\xi^{-2} \nu(x)($ by $(3.10))=0$. Thus,

$$
0=\nu(y)=Q\left(b_{2}\right)+\xi^{2} Q\left(b_{3}\right)+\frac{\xi}{2} \phi\left(U_{b_{2}} b_{3}, b_{3}\right)-\frac{\xi}{4} \phi\left(b_{2}, b_{3}\right)^{2} .
$$

Setting $\xi=0$ gives $Q\left(b_{2}(0)\right)=0$, where $b_{2(0)}=\Sigma g_{i}(0) v_{i}$. As above, this implies that $b_{2}(0)=0$ and so $\xi \mid g_{i}(\xi)$ for all $i$. This is the desired contradiction. 
EXAMPLE 7.2. Suppose $\mathfrak{f}=F(\xi)$, where $\xi$ is transcendental over a field $F$, and suppose $\mathscr{B}=\mathcal{C} \otimes_{F} \mathfrak{f}$, where $\mathcal{C}$ is a central Jordan division algebra of degree 4 over $f$. Let $n$ be the generic norm on $\mathscr{B}$ and put $\left(\mathscr{Q},,^{-}\right)=C D(\Re, n, \xi)$. By Theorem 7.1, $\left(Q,,^{-}\right)$is a structurable division algebra. Now $\operatorname{dim}_{F}(C)=10,16$, or 28 and so $\operatorname{dim}_{f}(\mathbb{Q})=20,32$, or 56. If char $\mathfrak{k}=0$, the corresponding Lie algebras $\mathscr{K}\left(\mathbb{Q},,^{-}\right)$are relative rank 1 central simple Lie algebras of type $E_{6}, E_{7}$, or $E_{8}$, respectively. In fact, one can show that the indices of these algebras are ${ }^{2} E_{6,1}^{35}, E_{7,1}^{66}$, or $E_{8,1}^{133}$, respectively (using the notation of [19]).

8. Reduced algebras arising from the Cayley-Dickson process. Throughout this section, assume that $\Re$ is a separable Jordan algebra of degree 4 over $\mathfrak{f}, n$ and t are the generic norm and trace, respectively, on $\Re$, and $\mu \in \mathfrak{f}^{*}$. Let $\left(\mathfrak{Q},,^{-}\right)=C D(\mathscr{B}, n, \mu)$. Our goal is to give necessary and sufficient conditions for $\left(Q,,^{-}\right)$to be reduced when $\mathfrak{f}$ is infinite.

The multiplication and involution on $\mathscr{Q}=\mathscr{B} \oplus s_{0} \mathscr{B}$ are given by (6.3) and (6.4) respectively, where

$$
b^{\theta}=-b+\frac{1}{2} t(b) 1
$$

and hence

$$
t\left(b_{1}^{\theta}, b_{2}^{\theta}\right)=t\left(b_{1}, b_{2}\right)
$$

for $b, b_{1}, b_{2} \in \Re$. Put $\chi=\chi_{\left(\Theta_{,-}\right)}$and $\nu=\nu_{\left(\Theta_{,-}\right)}$. Then, by (6.8) and (6.9),

$$
\chi\left(b_{1}+s_{0} b_{2}, b_{3}+s_{0} b_{4}\right)=t\left(b_{1}, b_{3}\right)-\mu t\left(b_{2}, b_{4}\right)
$$

and

$$
\nu\left(b_{1}+s_{0} b_{2}\right)=n\left(b_{1}\right)+\mu^{2} n\left(b_{2}\right)+\frac{\mu}{2} t\left(U_{b_{1}} b_{2}, b_{2}\right)-\frac{\mu}{4} t\left(b_{1}, b_{2}\right)^{2} .
$$

Moreover, by Corollary 6.10, if $c \in \mathscr{B}$ is invertible, then

$$
P_{c}\left(b_{1}+s_{0} b_{2}\right)=U_{c} b_{1}+n(c) s_{0} U_{c}^{-1} b_{2} .
$$

Let $\lambda$ be transcendental over $f$ and put

$$
m_{b}(\lambda)=n(\lambda 1-b)=\sum_{i=0}^{4} m_{i}(b) \lambda^{i}
$$

for $b \in \Re$. Then, by (5.2), we have,

$$
\begin{aligned}
& m_{4}(b)=1 \\
& m_{3}(b)=-t(b), \\
& m_{2}(b)=\frac{1}{2}\left(t(b)^{2}-t\left(b^{2}\right)\right), \\
& m_{1}(b)=\frac{1}{6}\left(3 t(b) t\left(b^{2}\right)-2 t\left(b^{3}\right)-t(b)^{3}\right),
\end{aligned}
$$

and

$$
n(b)=m_{0}(b)=\frac{1}{24}\left(3 t\left(b^{2}\right)^{2}+8 t(b) t\left(b^{3}\right)-6 t\left(b^{4}\right)-6 t(b)^{2} t\left(b^{2}\right)+t(b)^{4}\right)
$$

We require some preliminary lemmas. 
Lemma 8.7. Suppose $b_{1}, b_{2} \in$ 沐 and $b_{1} b_{2}^{i}=b_{2} b_{1}^{i}=0$ for $i=1,2$ and 3. Then, (8.8)

$$
n\left(b_{1}+b_{2}\right)=n\left(b_{1}\right)+n\left(b_{2}\right)-t\left(b_{1}\right) m_{1}\left(b_{2}\right)-t\left(b_{2}\right) m_{1}\left(b_{1}\right)+m_{2}\left(b_{1}\right) m_{2}\left(b_{2}\right) \text {. }
$$

Proof. Let $b=b_{1}+b_{2}$. Since $\mathscr{G}$ is a Jordan algebra, we have $2(d a)(d c)+d^{2}(a c)$ $=a\left(d^{2} c\right)+2 d((d a) c)$ for $a, c, d \in \mathscr{B}$. Taking $a=c=b_{2}$ and $d=b_{1}$ gives $b_{1}^{2} b_{2}^{2}=0$. Hence, $b^{i}=b_{1}^{i}+b_{2}^{i}$ for $i=1,2,3$, and 4. Thus, using (8.6), we can obtain expressions for both sides of (8.8) in terms of $t\left(b_{j}^{i}\right), i=1,2,3,4, j=1,2$. These expressions turn out to be the same. We omit the details as they are straightforward.

LEMmA 8.9. Suppose $e \neq 0 \in \mathscr{B}$ is an idempotent. If $U_{e} \mathscr{G} \subseteq \mathfrak{f} e$, then $t(e)=1$. Moreover, if $t(e)=1$, then

$$
n(\alpha e+\beta(1-e))=\alpha \beta^{3}
$$

for $\alpha, \beta \in \mathfrak{k}$.

Proof. These facts are well known. For the proof, we may assume $f$ is algebraically closed. Then, the first statement follows from [4, Theorems 3.4.6(c) and 3.7.4]. For the last statement, suppose that $t(e)=1$. Then, by $(8.6), m_{i}(e)=0$ for $i=0,1,2$ and $m_{3}(e)=1$. Thus, for $\lambda, \delta \in \mathfrak{H}$,

$$
n(\lambda 1-\delta e)=\sum_{i=0}^{4} m_{i}(\delta e) \lambda^{i}=\lambda^{4}-\delta \lambda^{3} .
$$

Taking $\lambda=\beta$ and $\delta=\beta-\alpha$, we obtain (8.10).

LEMMA 8.11. Suppose $\mathfrak{H}$ is infinite and $b \neq 0 \in \Re$. Then there exists invertible $c \in \mathscr{B}$ such that $t\left(U_{c} b\right) \neq 0$.

Proof. Suppose the contrary. Then, $t\left(U_{c} b\right)=0$ for all $c \in$. for all invertible $c \in \mathscr{B}$. Thus, since the invertible elements of 9 are Zariski dense in $\because 3$ (since $f$ is infinite), $t\left(b, c^{2}\right)=0$ for all $c \in \mathscr{B}$. Linearizing gives $t(b, c d)=0$ for all $c, d \in \mathscr{B}$ and hence $b=0$.

THEOREM 8.12. Suppose 9 is a separable Jordan algebra of degree 4 over an infinite field $\mathfrak{k}, n$ is the generic norm on $\mathscr{B}$, and $\mu \in \mathfrak{H}^{*}$. Then, $C D(\mathscr{B}, n, \mu)$ is reduced if and only if

$$
\mu=n(b) \beta^{2}
$$

for some $b \in \mathscr{B}$ and $\beta \in \mathfrak{H}$.

Proof. Suppose first of all that (8.13) holds for some $b \in \mathfrak{G}$ and $\beta \in \mathfrak{f}$. By (8.4), $\nu(b)=n(b)$. Thus, by Theorem 4.6, $C D(\mathscr{B}, n, \mu)$ is reduced.

For the converse, suppose that $\left(Q,,^{-}\right)=C D(\mathscr{B}, n, \mu)$ is reduced. Thus, there exists a strictly regular element $f=b_{1}+s_{0} b_{2} \in \mathbb{Q}, b_{1}, b_{2} \in \mathscr{B}$. Now by Theorem 4.6,

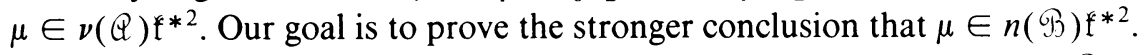

Suppose first of all that $b_{2}=0$. Then, $b_{1}$ is a nonzero element of $\mathscr{B}$ such that $U_{h} \mathscr{M} \subseteq \mathfrak{f} b_{1}$. Hence by Lemma 8.11, there exists $d \in \mathscr{B}$ such that $U_{d} \mathscr{G} \subseteq \mathfrak{f} d$ and $t(d) \neq 0$. But then by [10, Theorem 4.1], $d=\alpha e$, where $\alpha \in \mathfrak{l}^{*}$ and $e \neq 0 \in \mathscr{B}$ is an idempotent such that $U_{e} \mathscr{B} \subseteq \mathfrak{f} e$. By Lemma 8.9, $t(e)=1$. Thus, if we put $b=\mu e+$ $1-e$, we have $\mu=n(b)$ by (8.10). Therefore, we may assume $b_{2} \neq 0$. 
On the other hand, if $b_{1}=0$ then $\mu^{-1} L_{s_{0}} e=b_{2}$ is strictly regular. Therefore, by the argument in the previous paragraph, we may assume that $b_{1} \neq 0$.

By Lemma 8.11, the sets $\left\{c \in \mathscr{B} \mid n(c) \neq 0, t\left(U_{c} b_{1}\right) \neq 0\right\}$ and $\{c \in \mathscr{G} \mid n(c) \neq 0$, $\left.t\left(U_{c}^{-1} b_{2}\right) \neq 0\right\}$ are nonempty. Since these are Zariski open sets in $\mathscr{B}$ they have nonempty intersection. Thus, there exists invertible $c \in \mathscr{S}_{B}$ such that $t\left(U_{c} b_{1}\right) \neq 0$ and $t\left(U_{c}^{-1} b_{2}\right) \neq 0$. Thus, by (8.5), we may replace $f$ by $P_{c} f$ and assume $t\left(b_{1}\right) \neq 0$ and $t\left(b_{2}\right) \neq 0$.

Now $U_{f} 1=\delta f$ for some $\delta \in \mathfrak{k}$. But $U_{f} 1=2 f^{2}-\overline{f f}$ and so a straightforward calculation using (6.3), (6.4), (8.1), and (8.2) yields

$$
\begin{aligned}
U_{f} 1= & b_{1}^{2}+\mu\left(b_{2}^{2}-t\left(b_{2}\right) b_{2}+\frac{1}{2}\left(t\left(b_{2}\right)^{2}-t\left(b_{2}^{2}\right)\right) 1\right) \\
& +s_{0}\left(-2 b_{1} b_{2}+t\left(b_{1}\right) b_{2}+\frac{1}{2} t\left(b_{1}, b_{2}\right) l\right) .
\end{aligned}
$$

Thus, by (8.6), we have

$$
b_{1}^{2}+\mu\left(b_{2}^{2}-t\left(b_{2}\right) b_{2}+m_{2}\left(b_{2}\right) 1\right)=\delta b_{1}
$$

and

$$
-2 b_{1} b_{2}+t\left(b_{1}\right) b_{2}+\frac{1}{2} t\left(b_{1}, b_{2}\right) 1=\delta b_{2} .
$$

Applying $t$ to the second of these equations yields $t\left(b_{1}\right) t\left(b_{2}\right)=\delta t\left(b_{2}\right)$ and hence $\delta=t\left(b_{1}\right)$. Thus,

$$
\mu\left(b_{2}^{2}-t\left(b_{2}\right) b_{2}+m_{2}\left(b_{2}\right) 1\right)=-b_{1}^{2}+t\left(b_{1}\right) b_{1}
$$

and

$$
b_{1} b_{2}=\frac{1}{4} t\left(b_{1}, b_{2}\right) 1
$$

Applying $t$ to (8.14) gives

$$
m_{2}\left(b_{1}\right)=\mu m_{2}\left(b_{2}\right) .
$$

Now by identity $\left(\mathrm{B}_{3}\right)$ on p. 34 of $[\mathbf{1 0}]$, we have

$$
((a b) c) d+((a d) c) b+a((b d) c)=(a b)(c d)+(a c)(b d)+(a d)(b c)
$$

for $a, b, c, d \in \Re$. Putting $a=c=b_{1}$ and $b=d=b_{2}$ in this identity gives

$$
2\left(\left(b_{1} b_{2}\right) b_{1}\right) b_{2}+b_{1}\left(b_{2}^{2} b_{1}\right)=2\left(b_{1} b_{2}\right)^{2}+b_{1}^{2} b_{2}^{2} .
$$

In view of $(8.15)$, this implies that $b_{1}\left(b_{2}^{2} b_{1}\right)=b_{1}^{2} b_{2}^{2}$. Thus, $\left[b_{1}, b_{1}, b_{2}^{2}\right]=0$. Hence, by (8.14), $\left[b_{1}, b_{1}, \mu t\left(b_{2}\right) b_{2}\right]=0$. Since $\mu t\left(b_{2}\right) \neq 0$, we obtain $\left[b_{1}, b_{1}, b_{2}\right]=0$ and so, by (8.15),

$$
b_{1}^{2} b_{2}=\frac{1}{4} t\left(b_{1}, b_{2}\right) b_{1}
$$

Similarly,

$$
b_{1} b_{2}^{2}=\frac{1}{4} t\left(b_{1}, b_{2}\right) b_{2}
$$

Suppose next that $t\left(b_{1}, b_{2}\right) \neq 0$. Put $\gamma=\frac{1}{4} t\left(b_{1}, b_{2}\right)$. Then, (8.15) and (8.17) imply that $b_{1}$ is invertible and $b_{2}=\gamma b_{1}^{-1}$. Thus, $f=b_{1}+\gamma s_{0} b_{1}^{-1}$. Now $f$ is not invertible and so $\nu(f)=0$. But by (8.4),

$$
\nu(f)=n\left(b_{1}\right)+\mu^{2} \gamma^{4} n\left(b_{1}\right)^{-1}+2 \mu \gamma^{2}-4 \mu \gamma^{2}=n\left(b_{1}\right)^{-1}\left(n\left(b_{1}\right)-\mu \gamma^{2}\right)^{2} .
$$


Thus, $n\left(b_{1}\right)=\mu \gamma^{2}$ and so $\mu=n\left(b_{1}\right)\left(\gamma^{-1}\right)^{2}$ as required. Therefore, we may assume that $t\left(b_{1}, b_{2}\right)=0$.

By (8.15), (8.17), and (8.18), we have

$$
b_{1} b_{2}=b_{1}^{2} b_{2}=b_{1} b_{2}^{2}=0 .
$$

Multiplying (8.14) by $b_{2}$ gives

$$
b_{2}^{3}-t\left(b_{2}\right) b_{2}^{2}+m_{2}\left(b_{2}\right) b_{2}=0 .
$$

Also multiplying (8.14) by $b_{1}$ and using (8.16) gives

$$
b_{1}^{3}-t\left(b_{1}\right) b_{1}^{2}+m_{2}\left(b_{1}\right) b_{1}=0 .
$$

Hence by (8.19), (8.20) and (8.21), we have

$$
b_{1} b_{2}^{i}=b_{2} b_{1}^{i}=0, \quad i=1,2,3 .
$$

Next $U_{b_{1}} b_{2}=U_{b_{2}} b_{1}=0$ (by (8.19)) and so $n\left(b_{1}\right)=n\left(b_{2}\right)=0$. Also multiplying $(8.21)$ by $b_{1}$ gives $b_{1}^{4}-t\left(b_{1}\right) b_{1}^{3}+m_{2}\left(b_{1}\right) b_{1}^{2}=0$. On the other hand, $0=b_{1}^{4}-$ $t\left(b_{1}\right) b_{1}^{3}+m_{2}\left(b_{1}\right) b_{1}^{2}+m_{1}\left(b_{1}\right) b_{1}$, since $m_{0}\left(b_{1}\right)=n\left(b_{1}\right)=0$. Thus, $m_{1}\left(b_{1}\right)=0$ and similarly $m_{1}\left(b_{2}\right)=0$. Thus, by (8.8), we have $n\left(b_{1}+b_{2}\right)=m_{2}\left(b_{1}\right) m_{2}\left(b_{2}\right)=$ $\mu m_{2}\left(b_{2}\right)^{2}$. If $m_{2}\left(b_{2}\right) \neq 0$, this equation implies (8.13) with $b=b_{1}+b_{2}$ and $\beta=$ $m_{2}\left(b_{2}\right)^{-1}$. Thus, we may assume that $m_{2}\left(b_{2}\right)=0$. Therefore, $t\left(b_{2}\right)^{2}=t\left(b_{2}^{2}\right)$ and, by (8.20), $b_{2}^{3}=t\left(b_{2}\right) b_{2}^{2}$. Thus, if we put $e=t\left(b_{2}\right)^{-2} b_{2}^{2}$, we have $t(e)=1$ and $e^{2}=$ $t\left(b_{2}\right)^{-4} b_{2}^{4}=t\left(b_{2}\right)^{-2} b_{2}^{2}=e$. Hence, by $(8.10), n(\mu e+1-e)=\mu$.

We wish next to apply Theorem 8.12 to a special case. Suppose (요, ${ }^{-}$) is a central simple associative algebra with involution over $\mathfrak{f}^{\mathrm{s}}$ such that $\operatorname{dim}_{\mathrm{f}}$ of $=4,8$, or 16 and $\operatorname{dim}_{\mathrm{f}} \mathcal{H}\left(\mathscr{D},{ }^{-}\right)=3,4$, or 6 , respectively. Let $\mathbb{D}_{2}$ be the associative algebra of $2 \times 2$ matrices over $\mathscr{D}$ and define an involution $J_{1}$ on $\mathscr{D}_{2}$ by $J_{1}(x)=\bar{x}^{t}$. Put $\mathcal{E}=\mathcal{H}\left(\mathscr{L},,^{-}\right)$ and $\mathscr{B}=\mathscr{H}\left(\mathcal{Q}_{2}, J_{1}\right)$. Let $\overline{\mathfrak{f}}$ denote the algebraic closure of $\mathfrak{f}$. It follows from $[\mathbf{1 0}$, pp. 208-209] that $\left(\mathscr{O}_{\overline{\mathrm{f}}}^{-},{ }^{-}\right)$and $\left(\left(\mathcal{D}_{2}\right)_{\mathrm{f}}^{-},{ }^{-}\right)$can be identified, respectively, with the algebras of all $2 \times 2$ and $4 \times 4$ matrices over a composition algebra of dimension 1,2 , or 4 over $\bar{f}$, where the involution in each case is the conjugate transpose involution. Thus, by $[4$, Theorems 6.1 .7 and 6.3 .2$]$ and $[10, \S 6.4], \bigodot$ and $\Re$ are central simple Jordan algebras of degrees 2 and 4 , respectively. We denote the generic norms on $\mathscr{Q}_{2}, \mathcal{C}$, and $\mathscr{G}$ by $n_{\mathrm{OD}_{2}}, n_{\mathrm{e}}$ and $n_{\mathrm{SB}}$, respectively.

THEOREM 8.22. Suppose $\left.(\mathscr{Q}),^{-}\right)$is a central simple associative algebra with involution over $f$ such that $\operatorname{dim}_{\mathrm{f}} \mathscr{D}=4,8$, or 16 and $\operatorname{dim}_{\mathrm{f}} \mathcal{H}\left(\mathscr{D},,^{-}\right)=3,4$, or 6 , respectively. Let $\mathcal{e}=\mathcal{H}\left(\mathscr{\mathscr { L }},{ }^{-}\right)$and $\mathscr{B}=\mathcal{H}\left(\mathscr{Q}_{2}, J_{1}\right)$ and suppose $\mu \in \mathfrak{H}^{*}$. Then, $C D\left(\mathscr{T}, n_{\mathscr{B}}, \mu\right)$ is reduced if and only if there exist $c_{1}, c_{2} \in \mathcal{C}$ such that

$$
\mu=n_{\varrho}\left(c_{1}\right) n_{\varrho}\left(c_{2}\right) \text {. }
$$

Proof. To avoid confusion, we denote the associative product on $\mathbb{O}_{\text {and }} \mathscr{Q}_{2}$ by $\circ$. We require some preliminary facts. We claim that

$$
n_{\mathscr{B}}\left(\left[\begin{array}{ll}
c_{1} & 0 \\
0 & c_{2}
\end{array}\right]\right)=n_{\mathrm{C}}\left(c_{1}\right) n_{\mathrm{e}}\left(c_{2}\right) \text { and } n_{\mathrm{OP}_{2}}\left(\left[\begin{array}{ll}
1 & 0 \\
d & 1
\end{array}\right]\right)=1
$$

for $c_{1}, c_{2} \in \mathcal{E}$ and $d \in \mathscr{Q}$. We also claim that

$$
n_{\mathscr{D}_{2}} \mid \mathscr{B}=n_{\mathscr{B}}^{\prime},
$$


where $l=1$ if $\operatorname{dim}_{\mathrm{f}} \mathscr{D}=4$ and $l=2$ if $\operatorname{dim}_{\mathrm{f}} \mathscr{D}=8$ or 16 . To prove these claims we may assume that $\mathfrak{f}$ is algebraically closed and make the identifications described

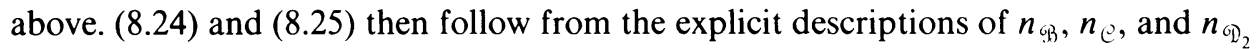
given in $[10, \S 6.4]$. We next claim that

$$
n_{\mathfrak{B}}\left(x \circ b \circ \bar{x}^{t}\right)=n_{\mathrm{OD}_{2}}(x)^{2 / l} n_{\mathfrak{C B}^{B}}(b)
$$

for $x \in \mathscr{D}_{2}, b \in \mathscr{B}$. For this we may assume that ${ }^{f}$ is infinite and $b$ is invertible. Then,

$$
n_{\mathscr{B}}\left(x \circ b \circ \bar{x}^{t}\right)^{l}=n_{\mathscr{Q}_{2}}\left(x \circ b \circ \bar{x}^{t}\right)=n_{\mathscr{Q ⿻}_{2}}(x) n_{\mathscr{Q}_{2}}(b) n_{\mathscr{Q}_{2}}\left(\bar{x}^{t}\right)=n_{\mathscr{P}_{2}}(x)^{2} n_{\mathscr{G}}(b)^{l} .
$$

Thus, $n_{\mathfrak{B}}\left(x \circ b \circ \bar{x}^{t}\right)= \pm n_{\mathscr{D}_{2}}(x)^{2 / l} n_{\mathfrak{G ̆}_{\mathfrak{B}}}(b)$. A Zariski argument as on [10, p. 249] then completes the proof of $(8.26)$.

We can now prove the theorem. Let $\left(Q,^{-}\right)=C D\left(\mathscr{B}, n_{\mathfrak{G}}, \mu\right)$. Suppose first of all that (8.23) holds. Then, by (8.24), $\mu=n_{\mathscr{B}}(b)$ for some $b \in \Re$. Hence, by (6.9), $\mu=\nu_{(\Theta,-}(b)$. Thus, by Theorem 4.6, $\left(\mathbb{Q},{ }^{-}\right)$is reduced.

Conversely, suppose that $\left(\mathscr{Q},{ }^{-}\right)$is reduced. Suppose first of all that $\mathcal{C}$ is not a division algebra. Then, $\mathcal{C}$ contains an idempotent $e \neq 0,1$ [10, Lemma 4.1]. But then denoting the generic trace on $e$ by $t_{\mathcal{C}}$, we have $t_{\mathcal{C}}(e)=1$ [10, Corollary 6.1]. But then if $c=\mu e+1-e$, we have $n_{\mathfrak{C}}(c)=\frac{1}{2}\left(t_{\mathfrak{C}}(c)^{2}-t_{\mathfrak{C}}\left(c^{2}\right)\right.$ ) (since $C$ has degree 2) $=$ $\frac{1}{2}\left((\mu+1)^{2}-\left(\mu^{2}+1\right)\right)=\mu$ and so we have (8.23).

Suppose next that $e$ is a division algebra. Thus, by [4, Lemma 6.2.3], ( $\left.\mathcal{D},^{-}\right)$is a central division algebra with involution or $\left(\mathscr{D},,^{-}\right)$is the direct sum of a central division algebra of dimension 4 and its opposite algebra together with the exchange involution. It follows (for later use) that every nonzero element of $\delta\left(\mathscr{D},,^{-}\right)$is invertible. Also it follows that $f$ is infinite and so we may apply Theorem 8.12 to conclude that $\mu=n(b) \beta^{2}$ for some $b \in \mathscr{B}$ and $\beta \in \mathfrak{f}$. Then,

$$
b=\left[\begin{array}{ll}
c_{1} & \bar{d}_{1} \\
d_{1} & c_{2}
\end{array}\right]
$$

where $c_{1}, c_{2} \in \mathcal{C}, d_{1} \in \mathcal{D}$.

Assume first the $c_{1} \neq 0$. By (8.24) and (8.26), we may replace $b$ by $x \circ b \circ \bar{x}^{t}$, where

$$
x=\left[\begin{array}{cc}
1 & 0 \\
-d \circ c_{1}^{-1} & 1
\end{array}\right] .
$$

Hence, we may assume that $d_{1}=0$. Thus,

$$
\mu=n_{\mathscr{B}}(b) \beta^{2}=n_{\bigodot}\left(c_{1}\right) n_{\bigodot}\left(c_{2}\right) \beta^{2}=n_{\bigodot}\left(\beta c_{1}\right) n_{\bigodot}\left(c_{2}\right)
$$

as required. A similar argument works if $c_{2} \neq 0$.

Finally, assume that $c_{1}=c_{2}=0$. Since $b \neq 0$, we have $d_{1} \neq 0$. If $x=\left[\begin{array}{l}1 \\ d \\ 1_{1}\end{array}\right]$ and $d \in \mathscr{D}$, then $x \circ b \circ \bar{x}^{t}$ has 2,2 entry $d_{1} \circ \bar{d}+d \circ \bar{d}_{1}$. If this entry is nonzero for some $d \in \mathcal{D}$, we are done by the previous paragraph. It suffices then to show that $d_{1} \circ \bar{d}+d \circ \bar{d}_{1} \neq 0$ for some $d \in \mathscr{D}$. If $d_{1} \notin \delta\left(\mathscr{D},{ }^{-}\right)$, we may take $d=1$. If $d_{1} \in$ $\delta\left(\mathscr{Q},{ }^{-}\right)$, then $d_{1}$ is invertible and so we may take $d=d_{1}$. 
EXAMPLE 8.29. Suppose $\mathfrak{k}=F(\xi)$, where $\xi$ is transcendental over a field $F$. Suppose $\left.(\mathscr{Q}),^{-}\right)=\left(\varepsilon \otimes_{F}{ }^{*},{ }^{-}\right)$, where $\left(\mathscr{E},{ }^{-}\right)$is a central simple associative algebra with involution over $F$ such that $\operatorname{dim}_{F} \mathcal{E}=4,8$, or 16 , $\operatorname{dim}_{F} \mathcal{H}\left(\mathcal{E},{ }^{-}\right)=3,4$, or 6 , respectively, and $\mathcal{H}\left(\mathcal{E},{ }^{-}\right)$is a Jordan division algebra. Let $\left.\mathcal{C}=\mathcal{H}(\mathscr{Q}),^{-}\right)$and $\mathscr{T}=\mathcal{H}\left(\mathscr{Q}_{2}, J_{1}\right)$. It is easy to show using an argument similar to the proof of Lemma 9.1 of [10] that there do not exist $c_{1}, c_{2} \in \mathcal{e}$ so that $\xi=n_{\mathrm{e}}\left(c_{1}\right) n_{\mathrm{e}}\left(c_{2}\right)$. Hence, by Theorem 8.22 , $C D\left(\mathscr{G}, n_{\bullet \rightarrow}, \xi\right)$ is not reduced. On the other hand $\mathscr{B}$ is not a division algebra and so, by Corollary $6.10, C D\left(\Re, n_{B B}, \xi\right)$ is not a division algebra. Thus the Cayley-Dickson process gives examples of nonreduced algebras that are not division algebras.

\section{REFERENCES}

1. B. N. Allison, A class of nonassociative algebras with involution containing the class of Jordan algebras, Math. Ann. 237 (1978), 133-156.

2. Models of isotropic Lie algebras, Comm. Algebra 7 (1979), 1835-1875.

3. B. N. Allison and W. Hein, Isotopes of some nonassociative algebras with involution, J. Algebra 69 (1981), 120-142.

4. H. Braun and M. Koecher, Jordan-algebren, Springer, New York, 1966.

5. R. B. Brown, A new type of nonassociative algebra, Proc. Nat. Acad. Sci. U.S.A. 50 (1963), 947-949.

6. , Groups of type $E_{7}$, J. Reine Angew. Math. 236 (1969), 79-102.

7. J. C. Ferrar, Strictly regular elements in Freudenthal triple systems, Trans. Amer. Math. Soc. 174 (1972), 313-331.

8. H. Freudenthal, Sur le groupe exceptionnel $E_{7}$, Nederl. Akad. Wetensch. Proc. Ser. A $56=$ Indag. Math. 15 (1953), 81-89.

9. N. Jacobson, Lie algebras, Interscience Tracts in Pure and Appl. Math., no. 10, Interscience, New York, 1962.

10. Structure and representations of Jordan algebras, Amer. Math. Soc. Colloq. Publ., vol. 39, Amer. Math. Soc., Providence, R. I., 1968.

11. I. L. Kantor, Models of exceptional Lie algehras, Soviet Math. Dokl. 14 (1973), 254-258.

12. K. McCrimmon, Norms and noncommutative Jordan algebras, Pacific J. Math. 15 (1965), 925-956.

13. A prooof of Schafer's conjecture for infinite dimensional forms admitting composition, J. Algebra 5 (1967), 72-83

14. K. Meyberg, Ein Theorie der Freudenthalschen Triplesysteme. I, II, Nederl. Akad. Wetensch. Proc. Ser. A 71 = Indag. Math. 30 (1968), 162-174, 175-190.

15. R. Schafer, Introduction to nonassociative algebras, Academic Press, New York, 1966.

16. G. B. Seligman, Rational methods in Lie algebras, Marcel Dekker, New York and Basel, 1976.

17. T. A. Springer, On a class of Jordan algebras, Nederl Akad. Wetensch. Proc. Ser. A 62 = Indag. Math. 21 (1959), 254-264.

18. Characterization of a class of cubic forms, Nederl. Akad. Wetensch Proc. Ser. A $65=$ Indag. Math. 24 (1962), 259-265.

19. J. Tits, Classification of algebraic semisimple groups, Proc. Sympos. Pure Math., vol. 9, Amer. Math. Soc., Providence, R. I., 1966, pp. 33-62.

Department of Mathematics, University of Alberta, Edmonton, Alberta T6G 2Gl, Canada

Department of Mathematics, University of Virginia, Charlottesville, Virginia 22903 